\title{
LINEARIZED STABILITY OF EXTREME SHOCK PROFILES IN SYSTEMS OF CONSERVATION LAWS WITH VISCOSITY ${ }^{1}$
}

BY

\author{
ROBERT L. PEGO ${ }^{2}$
}

\begin{abstract}
For a genuinely nonlinear hyperbolic system of conservation laws with added artificial viscosity, $u_{t}+f(u)_{x}=\varepsilon u_{x x}$, we prove that traveling wave profiles for small amplitude extreme shocks (the slowest and fastest) are linearly stable to perturbations in initial data chosen from certain spaces with weighted norm; i.e., we show that the spectrum of the linearized equation lies strictly in the left-half plane, except for a simple eigenvalue at the origin (due to phase translations of the profile). The weight $e^{c x}$ is used in components transverse to the profile, where, for an extreme shock, the linearized equation is dominated by unidirectional convection.
\end{abstract}

1. Introduction. In this paper we demonstrate the stability, in a linearized sense, of viscous shock profiles for small amplitude extreme shock waves of a hyperbolic system of conservation laws,

$$
u_{t}+f(u)_{x}=0, \quad u \in \mathbf{R}^{m} .
$$

The shock profiles are traveling wave solutions of an associated parabolic system obtained by adding "artificial viscosity",

$$
u_{t}+f(u)_{x}=\mu u_{x x} .
$$

For $\mu>0$ fixed, we exhibit a class of perturbations, determined by a weighted norm, with respect to which the profiles satisfy a linearized stability criterion put forth by Sattinger (1976).

We assume that the system (1.1) is strictly hyperbolic, so that the matrix $d f(u)$ has $m$ distinct real eigenvalues

$$
\lambda_{1}(u)<\cdots<\lambda_{m}(u)
$$

with corresponding right and left eigenvectors $r_{k}(u), l_{k}(u)$ for $k=1$ to $m$, with $l_{i} \cdot r_{j}=\delta_{i j}$. A $k$-shock for the system (1.1) is a two-valued weak solution

$$
u(x, t)= \begin{cases}u_{L}, & x<s t, \\ u_{R}, & x>s t,\end{cases}
$$

Received by the editors August 23, 1982 and, in revised form, December 16, 1982. This material was presented at the AMS Summer Research Conference on Nonlinear Partial Differential Equations in Durham, New Hampshire, June 20-26, 1982.

1980 Mathematics Subject Classification. Primary 35K55, 35B35; Secondary 35L65.

Key words and phrases. Conservation laws, viscosity, nonlinear parabolic systems, shock profiles, traveling waves, stability, Burgers' equation, weighted norms

${ }^{1}$ This paper is part of the author's doctoral dissertation, written under the direction of Professor Andrew J. Majda, at the University of California, Berkeley.

${ }^{2}$ Sponsored by the United States Army under Contract No. DAAG29-80-C-0041. This material is based upon work supported by the National Science Foundation under Grant No. MCS-7927062. Mod. 2. 
where $u_{L}$ and $u_{R}$ must satisfy the Rankine-Hugoniot jump conditions

$$
f\left(u_{L}\right)-f\left(u_{R}\right)-s\left(u_{L}-u_{R}\right)=0
$$

and the strict entropy inequalities

$$
\lambda_{k}\left(u_{L}\right)>s>\lambda_{k}\left(u_{R}\right), \quad \lambda_{k+1}\left(u_{R}\right)>s>\lambda_{k-1}\left(u_{L}\right) .
$$

Here $\lambda_{k}(u)$ is assumed to be genuinely nonlinear, that is $\nabla \lambda_{k} \cdot r_{k}(u) \neq 0$. We normalize $r_{k}$ so that $\nabla \lambda_{k} \cdot r_{k}(u) \equiv 1$. With $u_{R}$ fixed, a one parameter family of $k$-shocks exists, with $u_{L}=\tilde{u}^{k}(\varepsilon)$ and $s=\lambda_{k}\left(u_{R}\right)+\frac{1}{2} \varepsilon$ for $\varepsilon>0$ small, satisfying

$$
\tilde{u}^{k}(0)=u_{R}, \quad \frac{d \tilde{u}^{k}}{d \varepsilon}(0)=r_{k}\left(u_{R}\right) .
$$

An elegant proof of this fact may be found in Conlon (1980). Also see Lax (1957).

A viscous shock profile for a given $k$-shock (1.3) is a smooth traveling wave solution $\phi((x-s t) / \mu)$ of the system (1.2) such that $\phi(\xi) \rightarrow u_{L}$ as $\xi \rightarrow-\infty, \phi(\xi) \rightarrow u_{R}$ as $\xi \rightarrow+\infty$. The profile $\phi(\xi)$ is a solution of a system of ODE's,

$$
\phi_{\xi}=f(\phi)-f\left(u_{R}\right)-s\left(\phi-u_{R}\right),
$$

joining the two rest point $u_{R}$ and $u_{L}$. In the present situation, Foy (1964) proved that shock profiles $\phi(\xi ; \varepsilon)$ exist for small amplitude $k$-shocks (with $s=\lambda_{k}\left(u_{R}\right)+\frac{1}{2} \varepsilon$ ). His proof gives an asymptotic description of the profile as $\varepsilon \rightarrow 0$, showing that to second order in $\varepsilon$, the profile matches a properly scaled hyperbolic tangent profile for the scalar Burgers' equation, $u_{t}+u u_{x}=u_{x x}$, along the eigenvector $r_{k}\left(u_{R}\right)$.

We are concerned with the asymptotic stability of the solution $\phi((x-s t) / \mu ; \varepsilon)$ to perturbations in the initial data for a fixed $\mu>0$. Introduce the traveling coordinate $\xi=(x-s t) / \mu$ and scale $t$ by $t^{\prime}=t / \mu$. Then (1.2) becomes

$$
u_{t}=u_{\xi \xi}-(d f(u)-s) u_{\xi} .
$$

The profile $\phi(\xi ; \varepsilon)$ is a stationary solution of this equation. But so is $\phi(\xi+\gamma ; \varepsilon)$ for any phase shift $\gamma$. The best sort of stability one can expect in this situation is so-called orbital asymptotic stability, which means that initial data of the form $u(\xi, 0)=\phi(\xi)+u_{0}(\xi)$ yield decay of the form

$$
u(\xi, t)-\phi(\xi+\gamma) \rightarrow 0, \quad \text { as } t \rightarrow \infty
$$

for some phase shift $\gamma$, if $u_{0}$ is small.

The approach we follow is to examine the linearized stability of the profile $\phi$. Writing $u(\xi, t)=\phi(\xi)+v(\xi, t)$, the perturbation $v$ satisfies a nonlinear evolution equation

$$
v_{t}=e(v) .
$$

Linearizing this equation at $v=0$ (taking a formal Gâteaux derivative) we get

$$
v_{t}=\mathcal{L}^{\prime} v=v_{\xi \xi}-((d f(\phi)-s) v)_{\xi}-\left(d^{2} f(\phi) \phi_{\xi}\right) v .
$$

Typically, if one can show that the spectrum of $\mathcal{L}^{\prime}$ is contained strictly in the left-half plane, so solutions of the linearized equation decay exponentially, then one can show that small solutions of the nonlinear equation also decay exponentially. 
However, because the phase of the profile $\phi$ can be shifted an arbitrary amount, the derivative $\phi_{\xi}$ is a null function for $\mathcal{L}^{\prime}$, so 0 is in the spectrum of $\mathcal{L}^{\prime}$. This situation is typical for traveling waves in general, which arise in many contexts (e.g., nerve impulses, traveling fronts for reaction-diffusion systems). In a broad setting, Sattinger (1976) showed nevertheless that if 0 is a simple eigenvalue of $\mathcal{L}^{\prime}$, and if the rest of the spectrum of $\varrho^{\prime}$ on a suitable function space is contained strictly in the left-half plane, then indeed one obtains orbital asymptotic stability for the traveling wave $\phi$ with exponential adjustment. Actually, he required in addition that the resolvent $\left(\lambda-\mathcal{L}^{\prime}\right)^{-1}$ satisfy a certain asymptotic estimate. In our Appendix we show that this estimate is automatically valid for the systems he considers.

An important feature of Sattinger's analysis is the use of spatially weighted norms for function spaces to "push" the essential spectrum (the spectrum aside from isolated points of finite multiplicity) of $\mathcal{L}^{\prime}$ to the left. It may be verified, in fact, that the essential spectrum of our $L^{\prime}$ from (1.6) on unweighted $L^{p}$ spaces includes the origin (see the treatment of the essential spectrum in Henry (1981), appendix to Chapter 5). Our main result is to exhibit, for $\varepsilon$ sufficiently small and $k=1$ or $m$, a weighted space on which $\mathscr{L}^{\prime}$ satisfies Sattinger's linearized stability criteria. Unfortunately, our result does not immediately yield nonlinear stability by Sattinger's theorem, for our weight fails to satisfy two of Sattinger's hypotheses. In particular, (a) the weight is not a scalar function, and (b) it is not bounded below in some components, so the nonlinear terms in (1.5) may fail to be continuous on the weighted space. (In a supplement, we describe the spectrum on unweighted spaces.)

Our main result only applies to the extreme shock profiles, for which $k=1$ or $m$. Our approach is to try to decouple, to second order in $\varepsilon$, the part of the linearized equation (1.6) along $r_{k}\left(u_{R}\right)$ from the components along $r_{j}\left(u_{R}\right), j \neq k$. Because of Foy's result, the part along $r_{k}$ consists mainly of Burgers' equation linearized about its scalar profile $\left(1-\tanh \frac{1}{2} x\right)$. For that equation the weight $\left(\cosh \frac{1}{2} x\right)$ is standard. The components along $r_{j}$ for $j \neq k$ are dominated as $\varepsilon \rightarrow 0$ by convective terms $-\left(\lambda_{j}-\lambda_{k}\right) v_{\xi}^{j}$. For an extreme shock these terms convect in the same direction. Then these components may all be weighted the same to achieve exponential decay, which improves as $\varepsilon \rightarrow 0$ and permits the elimination of nonvanishing "cross-terms" coupling the $r_{k}$ and $r_{j}$ components together.

Let us now state our main result for 1-shock profiles ( $m$-shock profiles may be treated by space reversal, $x \rightarrow-x)$. By a linear change of coordinates, we may assume

$$
d f\left(u_{R}\right)=\operatorname{diag}\left(\lambda_{1}\left(u_{R}\right), \ldots, \lambda_{m}\left(u_{R}\right)\right) .
$$

We introduce the following spaces of functions with weighted norms. For $c>0$, $1 \leqslant p<\infty$, define

$$
\left(L^{p}\right)_{c}^{m}=\left\{u: \mathbf{R} \rightarrow \mathbf{R}^{m} \mid u^{1} \cosh c x \in L^{p}, u^{j} e^{-c x} \in L^{p}, j=2, \ldots, m\right\}
$$

with norm

$$
\|u\|_{p, c}=\max \left\{\left\|u^{1} \cosh c x\right\|_{L^{p}},\left\|u^{j} e^{-c x}\right\|_{L^{p}}, j=2, \ldots, m\right\} .
$$


Define $\left(C_{u}\right)_{c}^{m}$ and \|\|$_{\infty, c}$ similarly, where $C_{u}$ is the space of bounded uniformly continuous functions on $\mathbf{R}$ under the sup norm.

Theorem 1.1. Fix $u_{R} \in \mathbf{R}^{m}$ and suppose $\lambda_{1}(u)$ is genuinely nonlinear. Consider the linearized equation (1.6), $v_{t}=\varrho^{\prime} v$, for the evolution of perturbations of the 1-shock profile $\phi(\xi ; \varepsilon)$. Fix $c, 0<c<\frac{1}{2}$. Then there exists $\beta, 0<\beta<c(1-c)$ such that if $\varepsilon$ is sufficiently small, the spectrum of $\varrho^{\prime}$ on any of the spaces $\left(L^{p}\right)_{\varepsilon c / 2}^{m}, 1 \leqslant p<\infty$, or $\left(C_{u}\right)_{\varepsilon c / 2}^{m}$, consists of a simple isolated eigenvalue at the origin and a part which lies in $a$ sector strictly contained in the left-half plane,

$$
S_{\alpha}\left(-\frac{1}{4} \varepsilon^{2} \beta\right)=\left\{\lambda \in \mathbf{C}\left|\operatorname{Re}\left(\lambda+\frac{1}{4} \varepsilon^{2} \beta\right) \leqslant-(\cos \alpha)\right| \lambda+\frac{1}{4} \varepsilon^{2} \beta \mid\right\},
$$

where $\alpha$ depends on $\varepsilon, 0<\alpha<\pi / 2$.

2. Scaling and transformation. The first steps in our proof will be: (a) to scale the variables with $\varepsilon$, using Foy's result on the asymptotic shape of the profile $\phi(\xi ; \varepsilon)$ as $\varepsilon \rightarrow 0$, so that the part of (1.6) corresponding to the linearized Burgers' equation appears invariant of $\varepsilon$ as $\varepsilon \rightarrow 0$; (b) to transform the scaled equation by introducing appropriate weight functions. Theorem 1.1 will thereby be reduced to an equivalent statement (Theorem 2.1) concerned with the spectrum of the scaled and transformed operator on an unweighted space.

Without loss of generality, assume that $u_{R}=0=f\left(u_{R}\right)$. Let us recall Foy's asymptotic description of the 1-shock profile: For $\varepsilon>0$ sufficiently small,

$$
\phi(\xi ; \varepsilon)=\frac{1}{2} \varepsilon \psi\left(\frac{1}{2} \varepsilon \xi ; \varepsilon\right)
$$

where $\psi(x ; \varepsilon)$ has the form

$$
\psi(x ; \varepsilon)=\phi^{B}(x) r_{1}(0)+\varepsilon \zeta(x ; \varepsilon),
$$

where $\phi^{B}(x)=1-\tanh \frac{1}{2} x$ and $\sup _{x}|\zeta(x ; \varepsilon)| \leqslant C$ independent of $\varepsilon$. (This estimate may be read out of Foy's proof with a little care.)

Consider the terms in (1.6). By Taylor's theorem,

$$
\begin{aligned}
d f(\phi(\xi)) & =d f(0)+d^{2} f(0) \phi(\xi)+\tilde{R}(\xi) \phi^{2}(\xi), \\
d^{2} f(\phi(\xi)) \phi_{\xi} & =d^{2} f(0) \phi_{\xi}+\tilde{S}(\xi) \phi(\xi) \phi_{\xi}(\xi),
\end{aligned}
$$

where

$$
\tilde{R}(\xi)=\int_{0}^{1}(1-t) d^{3} f(t \phi(\xi)) d t, \quad \tilde{S}(\xi)=\int_{0}^{1} d^{3} f(t \phi(\xi)) d t
$$

$\tilde{R}(\xi)$ and $\tilde{S}(\xi)$ are bounded in $\xi$, and for each $\xi$ represent a trilinear map taking a vector triple to a vector. The equality

$$
\left(\tilde{R} \phi^{2}\right)_{\xi}=\tilde{S} \phi \phi_{\xi}
$$

holds, where each side is a matrix.

Let us now introduce the following scaled variables (which, despite appearances, have nothing to do with the original ones).

$$
x=\frac{1}{2} \varepsilon \xi, \quad t^{\prime}=\frac{1}{4} \varepsilon^{2} t, \quad \frac{1}{2} \varepsilon u=v .
$$


Then

$$
v_{t}=\frac{\varepsilon^{3}}{8} u_{t^{\prime}}, \quad \bar{v}_{\xi}=\frac{\varepsilon^{2}}{4} u_{x}, \quad v_{\xi \xi}=\frac{\varepsilon^{3}}{8} u_{x x}
$$

so (1.6) becomes

$$
\begin{aligned}
u_{t}= & \tilde{L}^{\prime} u=u_{x x}-\left[\frac{d f-s}{\varepsilon / 2}+d^{2} f \psi(x)+\frac{1}{2} \varepsilon \tilde{R}\left(x / \frac{1}{2} \varepsilon\right) \psi(x)^{2}\right] u_{x} \\
& -\left[d^{2} f \psi_{x}(x)+\frac{1}{2} \varepsilon \tilde{S}\left(x / \frac{1}{2} \varepsilon\right) \psi(x) \psi_{x}(x)\right] u .
\end{aligned}
$$

Here the derivatives $d f$ and $d^{2} f$ are evaluated at $0\left(=u_{R}\right)$. Define the following matrix-valued functions:

$$
\begin{aligned}
R(x) & =d^{2} f \zeta(x)+\frac{1}{2} \tilde{R}\left(x / \frac{1}{2} \varepsilon\right) \psi(x)^{2}, \\
S(x) & =d^{2} f \zeta_{x}(x)+\frac{1}{2} \tilde{S}\left(x / \frac{1}{2} \varepsilon\right) \psi(x) \psi_{x}(x) .
\end{aligned}
$$

Note that $R_{x}=S$, and $R(x ; \varepsilon), S(x ; \varepsilon)$ are uniformly bounded independent of $\varepsilon$. We also define the matrix $A=\left(a_{j}^{i}\right)$ by

$$
A=d^{2} f(0) r_{1}(0), \quad \text { so } a_{j}^{i}=l_{i} \cdot d^{2} f\left(r_{1}, r_{j}\right) \text {. }
$$

It is true that $a_{1}^{1}=\nabla \lambda_{1} \cdot r_{1}(0)=1$ (see Lax, 1957). Now (2.1) may be written in a slightly more coherent form:

$$
\tilde{\mathcal{L}}^{\prime} u=u_{x x}-\left[(d f-s) / \frac{1}{2} \varepsilon+\phi^{B}(x) A+\varepsilon R(x)\right] u_{x}-\left[\phi_{x}^{B}(x) A+\varepsilon R_{x}(x)\right] u .
$$

Observe that $d f-s=\operatorname{diag}\left(\left(\lambda_{i}-s\right), i=1, \ldots, m\right)$ and that $\left(\lambda_{1}-s\right) / \frac{1}{2} \varepsilon=-1$ by definition. Let us write (2.2) in a convenient block form. Write

$$
u=\left(\begin{array}{c}
u^{1} \\
\tilde{u}
\end{array}\right), \quad \text { where } \tilde{u}=\left(u^{2}, \ldots, u^{m}\right)^{t} .
$$

Relative to this decomposition, also write

$$
\frac{d f-s}{\varepsilon / 2}=\left[\begin{array}{cc}
-1 & 0 \\
0 & \Lambda / \frac{1}{2} \varepsilon
\end{array}\right], \quad A=\left[\begin{array}{cc}
1 & a^{1} \\
a_{1} & \tilde{A}
\end{array}\right], \quad R(x)=\left[\begin{array}{cc}
R_{1}^{1} & R^{1} . \\
R_{1} & \tilde{R}
\end{array}\right],
$$

where $\Lambda$ is a diagonal matrix with positive entries $\lambda_{2}-s, \ldots, \lambda_{m}-s$.

In block form, then, (2.2) is written

$$
\tilde{\mathcal{L}}^{\prime}\left(\begin{array}{c}
u^{1} \\
\tilde{u}
\end{array}\right)=\left[\begin{array}{cc}
L^{1} & M^{1} \\
\tilde{M} & \tilde{L}
\end{array}\right]\left(\begin{array}{c}
u^{1} \\
\tilde{u}
\end{array}\right)
$$

where the matrix entries are operators defined by

$$
\begin{array}{ll}
L^{1} u^{1}=L^{B} u^{1}-\varepsilon\left[R_{1}^{1} u^{1}\right]_{x}, & M^{1} \tilde{u}=-\left[\left(\phi^{B} a^{1}+\varepsilon R^{1}\right) \tilde{u}\right]_{x}, \\
\tilde{M} u^{1}=-\left[\left(\phi^{B} a_{1}+\varepsilon R_{1}\right) u^{1}\right]_{x}, & \tilde{L} \tilde{u}=L^{T} \tilde{u}-\left[\left(\phi^{B} \tilde{A}+\varepsilon \tilde{R}\right) \tilde{u}\right]_{x},
\end{array}
$$

and the dominant operators $L^{B}$ and $L^{T}$ are defined by

$$
L^{B} u^{1}=u_{x x}^{1}+\left(1-\phi^{B}(x)\right) u_{x}^{1}-\phi_{x}^{B}(x) u^{1}, \quad L^{T} \tilde{u}=\tilde{u}_{x x}-\left(\Lambda / \frac{1}{2} \varepsilon\right) \tilde{u}_{x} .
$$

This completes the appropriate scaling of the variables as $\varepsilon \rightarrow 0$. The operator $L^{B}$ is the operator that would be obtained by linearizing Burgers' equation $u_{t}+u u_{x}=$ $u_{x x}$ about the traveling wave solution $\phi^{B}(x-t)$ in traveling coordinates. It is well 
known (Peletier, 1971; Sattinger, 1976) that considered on a weighted function space with weight $w(x)=\cosh \frac{1}{2} x$, the operator $L^{B}$ satisfies the linearized stability criteria we are concerned with. (Briefly, the idea is that consideration of $L^{B}$ on the weighted space is equivalent to consideration of the transformed operator $w L^{B} w^{-1}$ on an unweighted space.) Roughly, what we would like to show, using arguments from perturbation theory for linear operators, is that $L^{B}$ "dominates" the first component of $\tilde{\mathcal{L}}^{\prime}$ and the other components only "contribute" spectrum strictly in the left-half plane.

Two observations are of primary importance. First, in general $a^{1} \neq 0$, so the first component of $\tilde{L}^{\prime}$ apparently cannot be considered a "small" perturbation of $L^{B}$. Second, observe that each component of the diagonal operator $L^{T}$ consists of just the Laplacian plus a translational term. The time evolution for this operator yields, in each component, solutions of the heat equation in a frame moving at constant velocity. No weight which is bounded below can yield exponential decay rates for such solutions (which are necessary if the spectrum of $L^{T}$ is to lie strictly in the left-half plane), for they decay to zero only algebraically in sup norm. However, in each component the solutions are being transported in the same direction at increasingly high velocity as $\varepsilon \rightarrow 0$ (since we are considering an extreme shock). Thus one may expect that if we use a weight $e^{c x}$ decaying in the direction of translation, we would obtain exponential decay for solutions of $\tilde{u}_{t}=L^{T} \tilde{u}$ at a rate which improves as $\varepsilon \rightarrow 0$. This should mean that the spectrum of $L^{T}$ moves further to the left as $\varepsilon \rightarrow 0$, so that for $\lambda$ fixed, one expects the resolvent $\left(\lambda-L^{T}\right)^{-1}$ to decay in norm as $\varepsilon \rightarrow 0$. Then the resolvent equation $\left(\lambda-\tilde{e}^{\prime}\right) u=f$ might be solved as if it were diagonally dominant, regardless of the coupling terms.

The discussion above completely describes our procedure, and motivates the following transformation: Fix $c>0$, and in the block form used above, define the matrix weight

$$
W_{c}(x)=\left[\begin{array}{cc}
\cosh c x & 0 \\
0 & e^{-c x}
\end{array}\right]
$$

Introduce the new dependent variable $z=W_{c} u$. Then the equation $u_{t}=\tilde{L}^{\prime} u$ is transformed into

$$
z_{t}=\tilde{\mathcal{L}}_{c}^{\prime} z=W_{c} \tilde{\mathcal{L}}^{\prime} W_{c}^{-1} z=\left[\begin{array}{cc}
w_{c} L^{1} w_{c}^{-1} & w_{c} M^{1} e^{c x} \\
e^{-c x} \tilde{M} w_{c}^{-1} & e^{-c x} \tilde{L} e^{c x}
\end{array}\right]\left(\begin{array}{c}
z^{1} \\
\tilde{z}
\end{array}\right)
$$

where $w_{c}(x)=\cosh c x$.

In this way, consideration of the spectrum of the original $L^{\prime}$ on the space $\left(L^{p}\right)_{\varepsilon c / 2}^{m}$ is equivalent to consideration of the spectrum of $\tilde{L}_{c}^{\prime}$ on the unweighted space $\left(L^{p}\right)_{0}^{m}$, except for a scaling factor. To verify the scaling, consider the following equivalent formulations of the resolvent equation:

$$
\left(\lambda-\tilde{\complement}_{c}^{\prime}\right) z=f(x) \text { in }\left(L^{p}\right)_{0}^{m} .
$$

Set $z=W_{c} u, f=W_{c} g$, and multiply by $W_{c}^{-1}$ to obtain

$$
\left(\lambda-\tilde{\mathfrak{L}}^{\prime}\right) u=g(x) \quad \text { in }\left(L^{p}\right)_{c}^{m} .
$$


Now set $v(\xi)=\frac{1}{2} \varepsilon u\left(\frac{1}{2} \varepsilon \xi\right)$. To verify the scaling, compute formally

$$
\mathcal{L}^{\prime} v(\xi)=v_{t}=\frac{\varepsilon^{3}}{8} u_{t^{\prime}}=\frac{\varepsilon^{3}}{8} \tilde{\mathcal{L}}^{\prime} u(x) .
$$

So, multiplying by $\varepsilon^{3} / 8$, we obtain

$$
\left(\frac{1}{4} \varepsilon^{2} \lambda-\mathcal{L}^{\prime}\right) v=h(\xi) \text { in }\left(L^{p}\right)_{\varepsilon c / 2}^{m},
$$

since $W_{c}\left(\frac{1}{2} \varepsilon \xi\right) h(\xi) \in\left(L^{p}\right)_{0}^{m}$. Therefore, $\lambda$ is in the resolvent set of $\tilde{\mathcal{L}}_{c}^{\prime}$ on $\left(L^{p}\right)_{0}^{m}$ if and only if $\frac{1}{4} \varepsilon^{2} \lambda$ is in the resolvent set of $\mathcal{L}^{\prime}$ on $\left(L^{p}\right)_{\varepsilon c / 2}^{m}$. Hence Theorem 1.1 is equivalent to

THeOREM 2.1. Fix $c, 0<c<\frac{1}{2}$. Then there exists $\beta, 0<\beta<c(1-c)$, such that if $\varepsilon$ is sufficiently small, then the spectrum of $\tilde{\mathcal{L}}_{c}^{\prime}$ on $\left(L^{p}\right)_{0}^{m}, 1 \leqslant p<\infty$, or $\left(C_{u}\right)_{0}^{m}$, consists of a simple isolated eigenvalue at the origin and a part which lies in the sector

$$
S_{\alpha}(-\beta)=\{\lambda \in \mathbf{C}|\operatorname{Re}(\lambda+\beta) \leqslant-(\cos \alpha)| \lambda+\beta \mid\}
$$

strictly contained in the left half plane, where $\alpha$ may depend on $\varepsilon$, with $0<\alpha<\pi / 2$.

The proof of this theorem has three main parts.

(1) Resolvent estimates for the operators $w_{c} L^{B} w_{c}^{-1}$ and $e^{-c x} L^{T} e^{c x}$.

(2) Verification that the first-order operator $w_{c} M^{1} e^{c x}$ has uniformly bounded coefficients. It suffices to show that

$$
\sup _{x}\left|\psi(x ; \varepsilon) e^{2 c x}\right|<C \quad \text { independent of } \varepsilon .
$$

This yields the strict requirement $c<\frac{1}{2}$.

(3) Solution of the resolvent equation for $\tilde{\mathfrak{L}}_{c}^{\prime}$ by block Gaussian elimination.

In the next two sections we treat (1). The estimate for $L^{T}$, improving as $\varepsilon \rightarrow 0$, is the key to the success of (3). Since $L^{T}$ is diagonal, it suffices to treat a scalar operator $u_{x x}-2 \mu u_{x}$, as $\mu \rightarrow \infty$. Our discussion of $L^{B}$ parallels Sattinger's treatment (1976) of the general scalar equation. The argument for (2) in $\$ 5$ consists of observing that the exponential decay obtained in a standard proof of the stable manifold theorem holds independent of $\varepsilon$ as $\varepsilon \rightarrow 0$. ( $\psi(x ; \varepsilon)$ is a solution of an ODE lying in the stable manifold of the point 0 .)

3. Weighted resolvent estimates for $u_{x x}-2 \mu u_{x}$ as $\mu \rightarrow \infty$. We consider the scalar differential operator

$$
L_{0} u=u_{x x}-2 \mu u_{x}
$$

and define

$$
L_{c} u=e^{-c x} L_{0} e^{c x} u=u_{x x}-(2 \mu-2 c) u_{x}-\left(2 \mu c-c^{2}\right) u .
$$

Observe that $L_{\mu} u=u_{x x}-\mu^{2} u$ is formally selfadjoint. We are concerned with the situation $\mu>c>0, c$ fixed, $\mu$ large. We seek norm estimates, improving as $\mu \rightarrow \infty$, for the operators $\left(\lambda-L_{c}\right)^{-1}$ and $(d / d x)\left(\lambda-L_{c}\right)^{-1}$ on $L^{p}$ and $C_{u}$, uniform for $\lambda$ in a fixed sector of $\mathbf{C}$.

The following result suffices for our purposes. We define

$$
S_{\alpha}(\beta)=\{\lambda \in \mathbf{C}|\operatorname{Re}(\lambda-\beta) \geqslant-(\cos \alpha)| \lambda-\beta \mid\},
$$

the sector with vertex $\beta \in \mathbf{R}$ and angle opening $2(\pi-\alpha)$ to the right. 
Proposition 3.1. Fix $\beta \in \mathbf{R}$ and $c>0$. For any $\delta>0$, if $\mu$ is sufficiently large, there exists $\alpha<\pi / 2$ positive, depending on $\mu$, so that

$$
\sup _{\lambda \in S_{\alpha}(\beta)}\left\|\left(\frac{d}{d x}\right)^{j}\left(\lambda-L_{c}\right)^{-1}\right\|<\delta
$$

for $j=0$ and 1 . The norm is the operator norm on $L^{p}$ (or $\left.C_{u}\right)$.

This proposition follows from estimates on the Green's function for $\lambda-L_{c}$, which is obtained by transforming through $L_{\mu}$. The resolvent equation for $L_{c}$ is $\left(\lambda-L_{c}\right) u$ $=f$. Now

$$
\left(\lambda-L_{c}\right)=e^{(\mu-c) x}\left(\lambda-L_{\mu}\right) e^{(c-\mu) x},
$$

so

$$
\left(\lambda-L_{\mu}\right) e^{(c-\mu) x} u=e^{(c-\mu) x} f .
$$

We therefore obtain the solution formula,

$$
\left(\lambda-L_{c}\right)^{-1} f(x)=\frac{1}{2 \gamma} \int_{-\infty}^{\infty} e^{-\gamma|x-y|+(\mu-c)(x-y)} f(y) d y,
$$

where $\gamma(\lambda)=\sqrt{\mu^{2}+\lambda}, \operatorname{Re} \gamma \geqslant 0$. Thus we define

$$
K_{\mu}(s, \lambda)=e^{-\gamma|s|+(\mu-c) s} / 2 \gamma,
$$

so $\left(\lambda-L_{c}\right)^{-1} f=K_{\mu}(\cdot, \lambda) * f$. When $\operatorname{Re} \gamma>\mu-c, K_{\mu}(\cdot, \lambda) \in L^{1}$ and it may be verified that the solution formula yields a bounded inverse for $\lambda-L_{c}$ on $L^{p}$, $1 \leqslant p<\infty$, on the domain

$$
\mathscr{D}\left(L_{c}\right)=\left\{f \in L^{p} \mid f^{\prime} \text { is absolutely continuous, } f^{\prime}, f^{\prime \prime} \in L^{p}\right\}
$$

(similarly for $C_{u}$ ). We omit the details, since a similar verification is carried out in the next section for the operator $w_{c} L^{B} w_{c}^{-1}$. We also find that

$$
\begin{aligned}
\frac{d}{d x}\left(\lambda-L_{c}\right)^{-1} f(x)=\frac{1}{2 \gamma}[(-\gamma+\mu & -c) \int_{-\infty}^{x} e^{(-\gamma+\mu-c)(x-y)} f(y) d y \\
& \left.+(\gamma+\mu-c) \int_{x}^{\infty} e^{(\gamma+\mu-c)(x-y)} f(y) d y\right] .
\end{aligned}
$$

So defining

$$
d K_{\mu}(s, \lambda)= \begin{cases}\frac{1}{2 \gamma}(-\gamma+\mu-c) e^{(-\gamma+\mu-c) s}, & \text { if } s>0, \\ \frac{1}{2 \gamma}(\gamma+\mu-c) e^{(\gamma+\mu-c) s}, & \text { if } s<0\end{cases}
$$

we have

$$
\frac{d}{d x}\left(\lambda-L_{c}\right)^{-1} f=d K_{\mu}(\cdot, \lambda) * f
$$

Using Young's inequality, $\|K * f\|_{L^{p}} \leqslant\|K\|_{L^{\prime}}\|f\|_{L^{p}}$, we find

$$
\left\|\left(\lambda-L_{c}\right)^{-1}\right\| \leqslant \frac{1}{2|\gamma|}\left(\frac{1}{\operatorname{Re} \gamma-(\mu-c)}+\frac{1}{\operatorname{Re} \gamma+(\mu-c)}\right)
$$


and

$$
\left\|\frac{d}{d x}\left(\lambda-L_{c}\right)^{-1}\right\| \leqslant \frac{1}{2|\gamma|}\left(\frac{|\gamma-(\mu-c)|}{\operatorname{Re} \gamma-(\mu-c)}+\frac{|\gamma+(\mu-c)|}{\operatorname{Re} \gamma+(\mu-c)}\right) .
$$

Proposition 3.2. Let $\gamma=\sqrt{\mu^{2}+\lambda}$ for $\lambda \in \mathbf{C}$. Then if $\mu>2 c, \alpha<\pi / 2$ may be chosen so that

(1) $|\gamma-(\mu-c)| /(\operatorname{Re} \gamma-(\mu-c)) \leqslant 3 \sqrt{\mu / c}$, if $\lambda \in S_{\alpha}(-\mu c)$,

(2) $|\gamma-(\mu-c)| \geqslant c / 4$, if $\lambda \in S_{\alpha}(-\mu c)$,

(3) $|\gamma+(\mu-c)| /(\operatorname{Re} \gamma+(\mu-c)) \leqslant \tan \frac{1}{2}(\pi-\alpha) \leqslant 2$, for $\lambda \in S_{\alpha}\left(-\mu^{2}\right)$.

These estimates yield, for $\mu>c, \lambda \in S_{\alpha}(-\mu c)$,

$$
\begin{gathered}
\left\|\frac{d}{d x}\left(\lambda-L_{c}\right)^{-1}\right\| \leqslant \frac{1}{2\left|\mu^{2}+\lambda\right|^{1 / 2}}(3 \sqrt{\mu / c}+2), \\
\left\|\left(\lambda-L_{c}\right)^{-1}\right\| \leqslant \frac{1}{2\left|\mu^{2}+\lambda\right|^{1 / 2}}\left(\frac{12}{c} \sqrt{\mu / c}+\frac{2}{\left|\mu^{2}+\lambda\right|^{1 / 2}}\right) .
\end{gathered}
$$

Proposition 3.1 follows, for $\left|\mu^{2}+\lambda\right| \geqslant\left(\beta+\mu^{2}\right) \sin \alpha$ if $\lambda \in S_{\alpha}(\beta)$, so the bounds above tend to zero uniformly for $\lambda \in S_{\alpha}(\beta)$ as $\mu \rightarrow \infty$ at the rate $\mu^{-1 / 2}$.

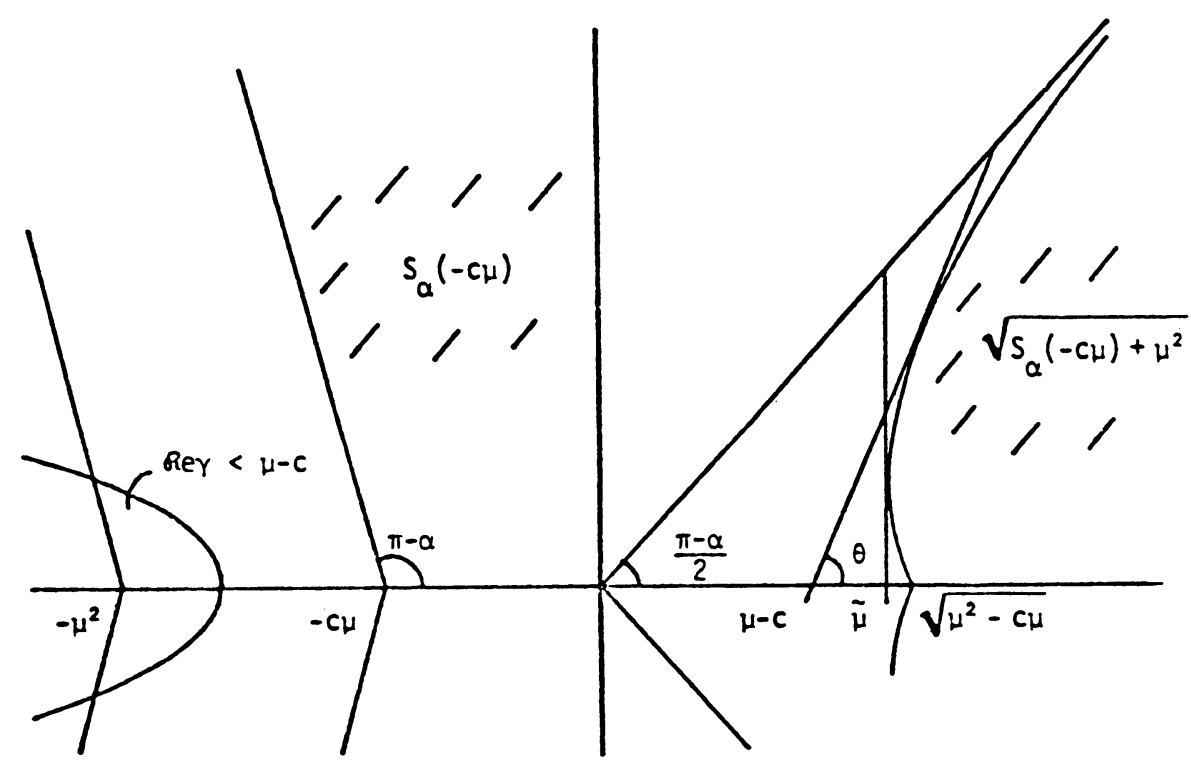

FIGURE 1. Action of the map $\lambda \rightarrow \sqrt{\mu^{2}+\lambda}$

Proof of Proposition 3.2. Figure 1 gives a "before and after" view of the complex plane under the map $\lambda \rightarrow \sqrt{\mu^{2}+\lambda}$. Part (3) of the proposition becomes obvious, and parts (1) and (2) are implied by the estimates
(a) $\sec \theta \leqslant 3 \sqrt{\mu / c}$ and
(b) $\tilde{\mu}>(\mu-c)+c / 4$. 
Calculation of $\tilde{\mu}$. Define

$$
\tilde{\mu}=\min _{\lambda \in S_{\alpha}(-c \mu)} \operatorname{Re} \sqrt{\mu^{2}+\lambda} .
$$

We introduce some notation. Set $\beta=\mu^{2}-c \mu, z=-\cos \alpha+i \sin \alpha, \kappa=\cos \alpha$, and $s=\sin \alpha$. For $t>0, \lambda=-\mu c+t z$ lies on the boundary of $S_{\alpha}(-c \mu)$ and $\gamma=\sqrt{\mu^{2}+\lambda}$ $=\sqrt{\beta+t z}$. We minimize $\operatorname{Re} \gamma(t)=\operatorname{Re} \sqrt{\beta+t z}$ with respect to $t$. Now $(\operatorname{Re} \gamma)^{2}$ $=\frac{1}{2}(\beta-\kappa t+n(t))$, where $n(t)=|\beta+t z|$. One calculates

$$
n^{\prime}(t)=\frac{t-\beta \kappa}{|\beta+t z|} \text {. }
$$

Then

$$
\left[(\operatorname{Re} \gamma)^{2}\right]^{\prime}=2(\operatorname{Re} \gamma)(\operatorname{Re} \gamma)^{\prime}=\frac{1}{2}\left(-\kappa+\frac{t-\beta \kappa}{n(t)}\right)=0
$$

only when $|\beta+t z| \kappa=t-\beta \kappa$, or

$$
\kappa^{2}\left(\beta^{2}-2 \beta \kappa t+t^{2}\right)=t^{2}-2 \beta \kappa t+\beta^{2} \kappa^{2}
$$

or, since we seek $t \neq 0, t\left(1-\kappa^{2}\right)=2 \beta \kappa\left(1-\kappa^{2}\right)$. Therefore, $t=2 \beta \kappa$, whence $(\tilde{\mu})^{2}=\beta s^{2}$, so

$$
\tilde{\mu}=\sin \alpha \sqrt{\mu^{2}-c \mu} .
$$

Now $\beta=(\mu-c)^{2}+c(\mu-c)$. If $\mu / c>2$, then $c /(\mu-c)<1$, and we have

$$
\sqrt{\beta}>(\mu-c)\left(1+(\sqrt{2}-1) \frac{c}{\mu-c}\right)>(\mu-c)+\frac{c}{3} .
$$

Hence we may choose $\alpha<\pi / 2$ depending on $\mu$ so that

$$
(\sin \alpha)(\mu-c+c / 3)>(\mu-c+c / 4) .
$$

Thus $\tilde{\mu}>(\mu-c)+c / 4$.

Estimate of $\sec \theta$. Define $\theta$ by

$$
\sec \theta=\max _{\lambda \in S_{\alpha}(-c \mu)} \frac{|\gamma-(\mu-c)|}{\operatorname{Re} \gamma-(\mu-c)} .
$$

Claim. If $\alpha=\pi / 2$, then $\sec ^{2} \theta=\mu / c+1$. Define

$$
h(t)=|\gamma(t)-(\mu-c)|=|\sqrt{\beta+t i}-(\mu-c)|, \quad g(t)=\operatorname{Re} \gamma(t)-(\mu-c),
$$

where $\beta=\mu^{2}-c \mu$ as above. We proceed to maximize $h^{2}(t) / g^{2}(t)$ with respect to $t$. This quantity is a maximum when $g^{2}\left(h^{2}\right)^{\prime}=h^{2}\left(g^{2}\right)^{\prime}$. We calculate (with $n(t)=$ $|\beta+t i|)$ :

$$
\begin{aligned}
h^{2}(t) & =|\beta+t i|-2(\mu-c) \operatorname{Re} \gamma+(\mu-c)^{2}, \\
g^{2}(t) & =\frac{1}{2}(\beta+n(t))-2(\mu-c) \operatorname{Re} \gamma+(\mu-c)^{2}, \\
(\operatorname{Re} \gamma)^{2 \prime} & =2 \operatorname{Re} \gamma(\operatorname{Re} \gamma)^{\prime}=\frac{1}{2} n^{\prime}(t), \\
\left(h^{2}\right)^{\prime} & =n^{\prime}(t)-2(\mu-c) n^{\prime}(t) / 4 \operatorname{Re} \gamma, \\
\left(g^{2}\right)^{\prime} & =\frac{1}{2} n^{\prime}(t)-2(\mu-c) n^{\prime}(t) / 4 \operatorname{Re} \gamma .
\end{aligned}
$$


Thus $h^{2} / g^{2}$ is critical when

$$
g^{2}(2 \operatorname{Re} \gamma-(\mu-c))=h^{2}(\operatorname{Re} \gamma-(\mu-c))=h^{2} g
$$

or

$$
g \operatorname{Re} \gamma=h^{2}-g^{2}
$$

Thus

$$
(\operatorname{Re} \gamma)^{2}-(\mu-c) \operatorname{Re} \gamma=\frac{1}{2}(n(t)-\beta)
$$

or

$$
\frac{1}{2}(\beta+n(t))-(\mu-c) \operatorname{Re} \gamma=\frac{1}{2}(n(t)-\beta),
$$

so $\beta=(\mu-c) \operatorname{Re} \gamma$. Therefore, $\mu=\operatorname{Re} \gamma$ at the critical point $t$. Squaring, we have $\mu^{2}=\frac{1}{2}(\beta+n(t)), n(t)=2 \mu^{2}-\beta$. Squaring again, $\beta^{2}+t^{2}=4 \mu^{4}-4 \mu^{2} \beta+\beta^{2}$, so $t^{2}=4 \mu^{2}\left(\mu^{2}-\beta\right)=4 \mu^{2}(\mu c)$. Therefore, the only positive critical point for $h^{2} / g^{2}$ is $t=2 \mu \sqrt{\mu c}$. We calculate the value of $h^{2} / g^{2}$ at this point.

$$
n(2 \mu \sqrt{\mu c})=\sqrt{\beta^{2}+4 \mu^{3} c}=\sqrt{\mu^{2}\left[(\mu-c)^{2}+4 \mu c\right]}=\mu(\mu+c) .
$$

Since $\operatorname{Re} \gamma(2 \mu \sqrt{\mu c})=\mu$, we have

$$
\begin{aligned}
\left.\frac{h^{2}}{g^{2}}\right|_{t=2 \mu \sqrt{\mu c}} & =\frac{\mu(\mu+c)-2(\mu-c) \mu+(\mu-c)^{2}}{(\mu(\mu-c)+\mu(\mu+c)) / 2-2(\mu-c) \mu+(\mu-c)^{2}} \\
& =\frac{c \mu+c^{2}}{c^{2}}=\frac{\mu}{c}+1 .
\end{aligned}
$$

At $t=0, h^{2} / g^{2}=1$, and for large $t, h^{2} / g^{2}<2$. Therefore, the maximum value of $h^{2} / g^{2}$ is $\mu / c+1$, establishing our claim for $\alpha=\pi / 2$.

To complete estimate (a) for $\sec \theta$, fix $\mu$, and observe that $h^{2} / g^{2}(t, \alpha)$, properly defined, is continuous in both variables so long as $\pi / 2-\alpha$ is so small that $g>c / 4$. Therefore, if $\pi / 2-\alpha$ is sufficiently small (depending on $\mu$ ), then

$$
\max _{0 \leqslant t \leqslant \infty} h^{2}(t, \alpha) / g^{2} \leqslant 9 \mu / c,
$$

establishing (1) of Proposition 3.2.

4. The resolvent set for $L^{B} u=u_{x x}+\left(\tanh \frac{1}{2} x\right) u_{x}+\left(\frac{1}{2} \operatorname{sech}^{2} \frac{1}{2} x\right) u$. In this section we discuss the resolvent of $L^{B}$ on the weighted spaces $\left(L^{p}\right)_{c}^{1}$ and $\left(C_{u}\right)_{c}^{1}$ with weight $w_{c}(x)=\cosh c x$. Equivalently, we study the operator $L_{c}^{B} \equiv w_{c} L^{B} w_{c}^{-1}$ on the spaces $L^{p}, 1 \leqslant p<\infty$, and $C_{u} . L^{B}$ may be obtained by linearizing Burgers' equation $u_{t}+u u_{x}=u_{x x}$ about the traveling wave solution $\phi(x)=1-\tanh \frac{1}{2} \xi$ in traveling coordinates. Our present analysis may be considered an extension of that of Sattinger (1976), whose results apply to the formally selfadjoint operator $L_{1 / 2}^{B}$. Our result is that if $0<c<\frac{1}{2}$, the spectrum of $L_{c}^{B}$ on the spaces $L^{p}$ and $C_{u}$ consists of a simple eigenvalue at the origin, a discrete set of eigenvalues in the interval $\left(-\frac{1}{4}, 0\right)$, and a part contained in the parabolic region

$$
\left\{\lambda \in \mathbf{C} \mid \operatorname{Re} \lambda \leqslant-c(1-c)-\left(\frac{\operatorname{Im} \lambda}{1-2 c}\right)^{2}\right\} .
$$


Because of the next section, our results for $c$ strictly less than $\frac{1}{2}$ are required in the last section. In conjunction with Sattinger's treatment of nonlinear stability, these results imply that the traveling wave solution of Burgers' equation is stable to sufficiently small perturbations which decay exponentially as $|x| \rightarrow \infty$, no matter what the rate. A similar result might be obtained for other types of scalar equations that Sattinger considers. For scalar conservation laws with viscosity however, a more general result may be found in Il'in and Oleinik (1960).

Our proof uses the transformation to the selfadjoint form $L_{1 / 2}^{B}$ to carry information about that well-studied operator (its Green's function, the location and multiplicity of its eigenvalues) over to analyze the nonselfadjoint $L_{c}^{B}$. Let us collect the facts we require about $L_{1 / 2}^{B}$, following the development of Sattinger for the most part.

A short calculation shows that

$$
L_{1 / 2}^{B} u=u_{x x}-\frac{1}{4}\left(1-2 \operatorname{sech}^{2} \frac{1}{2} x\right) u .
$$

Lemma 4.1. Define $\gamma(\lambda)=\sqrt{\lambda+1 / 4}, \operatorname{Re} \gamma \geqslant 0$. If $\gamma \neq 0$, the homogeneous equation $\left(L_{1 / 2}^{B}-\lambda\right) u=0$ has a system of solutions $\phi_{-}, \phi_{+}, \psi_{-}$, and $\psi_{+}(x, \lambda)$ with the following asymptotic properties.

$$
\begin{array}{lll}
\phi_{ \pm}=e^{ \pm \gamma x}(1+o(1)), & \phi_{ \pm}^{\prime}=e^{ \pm \gamma x}( \pm \gamma+o(1)) & \text { as } x \rightarrow \infty, \\
\psi_{ \pm}=e^{ \pm \gamma x}(1+o(1)), & \psi_{ \pm}^{\prime}=e^{ \pm \gamma x}( \pm \gamma+o(1)) & \text { as } x \rightarrow-\infty .
\end{array}
$$

These functions are single-valued analytic functions of $\lambda$ in the complex plane cut from $-\infty$ to $-\frac{1}{4}$ along the real axis.

Proof. This is a standard result on the asymptotic behavior of solutions to linear ODE's. To obtain $\phi_{-}$, seek a solution of the form $\phi_{-}=z_{-} e^{-\gamma x}$. Then $z_{-}$must satisfy the equation $z^{\prime \prime}-2 \gamma z_{-}^{\prime}+p z_{-}=0$, where $p(x)=\frac{1}{2} \operatorname{sech}^{2} \frac{1}{2} x$. Assuming that $z_{-}^{\prime} \rightarrow 0$ and $z_{-} \rightarrow 1$ as $x \rightarrow \infty$, integrate to get

$$
z_{-}^{\prime}(x)=\int_{x}^{\infty} e^{2 \gamma(x-s)} p z_{-}(s) d s, \quad z_{-}(x)=1-\int_{x}^{\infty}\left(\frac{1-e^{2 \gamma(x-s)}}{2 \gamma}\right) p z_{-}(s) d s .
$$

Choose $x_{0}$ so that

$$
\int_{x_{0}}^{\infty} p d s<\frac{1}{2}|\gamma|
$$

Then a bounded solution $z_{-}(x)$ may be obtained on the interval $\left[x_{0}, \infty\right)$ through successive approximations. This solution does indeed satisfy $z_{-} \rightarrow 1, z_{-}^{\prime} \rightarrow 0$ as $x \rightarrow \infty$, yielding the desired behavior for $\phi_{-}$.

To obtain $\phi_{+}$, set $\phi_{+}=z_{+} e^{\gamma x}$. Then we must have $z_{+}^{\prime \prime}+2 \gamma z_{+}^{\prime}+p z_{+}=0$. Set $z_{+}\left(x_{0}\right)=1, z_{+}^{\prime}\left(x_{0}\right)=0$. Then, integrating,

$$
z_{+}^{\prime}(x)=-\int_{x_{0}}^{x} e^{-2 \gamma(x-s)} p z_{+}(s) d s, \quad z_{+}(x)=1-\int_{x_{0}}^{x}\left(\frac{1-e^{-2 \gamma(x-s)}}{2 \gamma}\right) p z_{+}(s) d s .
$$


Our choice of $x_{0}$ ensures that this last equation may be solved for a bounded $z_{+}(x)$ by successive approximations on the interval $\left[x_{0}, \infty\right)$. This solution has a nonzero limit as $x \rightarrow \infty$, so we normalize $z_{+}$, making this limit 1 and obtain $\phi_{+}$with the desired asymptotic properties.

Because of symmetry, we may set $\psi_{ \pm}(x)=\phi_{\mp}(-x)$. Since $z_{+}$and $z_{-}(x, \gamma)$ are obtained by successive approximations, they are analytic in $\gamma$ in the right-half plane. They are therefore analytic functions of $\lambda$ in the complex plane cut from $-\infty$ to $-\frac{1}{4}$.

Each pair $\phi_{-}, \phi_{+}$or $\psi_{-}, \psi_{+}$clearly forms a basis of solutions to the homogeneous equation $\left(L_{1 / 2}^{B}-\lambda\right) u=0$. Define the Wronskian,

$$
W(\lambda)=\phi_{-} \psi_{+}^{\prime}-\phi_{-}^{\prime} \psi_{+}
$$

(it is independent of $x$ ). The homogeneous equation has a bounded solution precisely when either $\operatorname{Re} \gamma=0$ or $W(\lambda)=0$. If $\operatorname{Re} \gamma=0$ (so $\lambda \leqslant-\frac{1}{4}$ ), all solutions are bounded. If $W(\lambda)=0$ but $\operatorname{Re} \gamma>0$, then $\phi_{-}$is a multiple of $\psi_{+}$, which then spans the set of bounded solutions. Conversely, if $\operatorname{Re} \gamma>0$ and $W(\lambda) \neq 0$, then any solution bounded as $x \rightarrow \infty$ is a multiple of $\phi_{-}$, but $\phi_{-}=A \psi_{-}+B \psi_{+}$, where $A \neq 0$. So no solution can remain bounded for all $x$.

Claim. The spectrum of $L_{1 / 2}^{B}$ on $L^{p}$ or $C_{u}$ consists exactly of the zero set of $W(\lambda)$ plus the interval $\left(-\infty,-\frac{1}{4}\right]$. Clearly, these points are eigenvalues for $L_{1 / 2}^{B}$ on the space $C_{u}$. If $W(\lambda)=0$ and $\operatorname{Re} \gamma>0$, then the eigenfunction $\phi_{-}(x, \lambda)$ is in $L^{p}$ for all $p \geqslant 1$. If $\operatorname{Re} \gamma=0$, then all solutions of the homogeneous equation oscillate, and $\phi_{-}$ is bounded but not in $L^{p}$. It is then easy to verify that $\lambda$ is in the approximate point spectrum of $L_{1 / 2}^{B}$ on $L^{p}, 1 \leqslant p<\infty$. (Consider the sequence $u_{n}=j(x / n) \phi_{-}(x)$, where $j(x)=0$ for $|x| \geqslant 1, j(x)=1$ for $|x| \leqslant \frac{1}{2}$, and $j$ is smooth.) It will follow from our analysis below of the resolvent of $L_{c}^{B}$ that $\lambda$ is in the resolvent set for $L_{1 / 2}^{B}$ when $\operatorname{Re} \gamma>0$ and $W(\lambda) \neq 0$.

LEMMA 4.2. Let $\lambda$ be a zero of $W(\lambda)$ in the complex plane cut from $-\infty$ to $-\frac{1}{4}$ along the real axis. Then (a) $\lambda$ is real and isolated, and (b) $\lambda \leqslant 0$.

Proof. (a) $\phi_{-}(x, \lambda)$ decays exponentially as $x \rightarrow \pm \infty$, so defining

$$
(u, v)=\int_{-\infty}^{\infty} u(x) \bar{v}(x) d x
$$

we have

$$
\lambda\left(\phi_{-}, \phi_{-}\right)=\left(L_{1 / 2}^{B} \phi_{-}, \phi_{-}\right)=\left(\phi_{-}, L_{1 / 2}^{B} \phi_{-}\right)=\bar{\lambda}\left(\phi_{-}, \phi_{-}\right),
$$

so that $\lambda=\bar{\lambda} . W(\lambda)$ is analytic and not identically zero in $\mathbf{C} \backslash\left(-\infty,-\frac{1}{4}\right]$, so has isolated zeros.

(b) The function $\phi_{-}(x, 0)=\frac{1}{2} \operatorname{sech} \frac{1}{2} x$ is positive. $\left(L_{1 / 2}^{B}\right.$ kills a positive function because it came from linearization about a monotone traveling wave.) If $W(\lambda)=0$ for some $\lambda>0$, define

$$
u(x)=\phi_{-}(x, \lambda) / \phi_{-}(x, 0) .
$$

Then since $\gamma(\lambda)>\gamma(0), u(x) \rightarrow 0$ as $x \rightarrow \pm \infty$. Also, $u$ satisfies the equation

$$
u_{x x}+\left(\tanh \frac{1}{2} x+2 \frac{\phi_{-}^{\prime}(x, 0)}{\phi_{-}(x, 0)}\right) u_{x}-\lambda u=0 \text {. }
$$


By the maximum principle, it follows that $u=0$, for $u$ cannot have a positive maximum (at which $u_{x x} \leqslant 0, u_{x}=0, u>0$ ), nor a negative minimum. (This argument applies to both real and imaginary parts if $u$ is not real.) This contradicts the fact $u \neq 0$. So $W(\lambda) \neq 0$ for $\lambda>0$.

We turn now to construct a resolvent formula for $L_{c}^{B}$. Assume that $\operatorname{Re} \gamma>0$ and $W(\lambda) \neq 0$. The Green's function for $\lambda-L_{1 / 2}^{B}$ is

$$
K_{1 / 2}(x, y, \lambda)= \begin{cases}\frac{1}{W(\lambda)} \psi_{+}(x, \lambda) \phi_{-}(y, \lambda), & \text { for } x<y, \\ \frac{1}{W(\lambda)} \phi_{-}(x, \lambda) \psi_{+}(y, \lambda), & \text { for } x>y .\end{cases}
$$

The resolvent formula for $L_{1 / 2}^{B}$ is

$$
\left(\lambda-L_{1 / 2}^{B}\right)^{-1} f(x)=\int_{-\infty}^{\infty} K_{1 / 2}(x, y, \lambda) f(y) d y .
$$

Now formally,

$$
\left(\lambda-L_{c}^{B}\right)^{-1}=\left(\frac{w_{c}}{w_{1 / 2}}\right)\left(\lambda-L_{1 / 2}^{B}\right)^{-1}\left(\frac{w_{c}}{w_{1 / 2}}\right)^{-1} .
$$

This yields the resolvent formula

$$
\left(\lambda-L_{c}^{B}\right)^{-1} f(x)=\int_{-\infty}^{\infty} K_{c}(x, y, \lambda) f(y) d y,
$$

where

$$
K_{c}(x, y, \lambda)= \begin{cases}\frac{1}{W(\lambda)} \psi_{+}(x) \frac{\cosh c x}{\cosh x / 2} \cdot \phi_{-}(y) \frac{\cosh y / 2}{\cosh c y}, & \text { for } x<y, \\ \frac{1}{W(\lambda)} \phi_{-}(x) \frac{\cosh c x}{\cosh x / 2} \cdot \psi_{+}(y) \frac{\cosh y / 2}{\cosh c y}, & \text { for } x>y .\end{cases}
$$

The essential step in proving that $\lambda$ is in the resolvent set for $L_{c}^{B}$ on $L^{p}$ is to prove that the resolvent formula above yields a bounded operator on $L^{p}$. Here is a well-known sufficient condition that an integral operator be bounded on $L^{p}$.

LEMMA 4.3. Let $K(x, y)$ be measurable, with $\|K(x, \cdot)\|_{L^{1}} \leqslant C_{1}$ independent of $x$, and $\|K(\cdot, y)\|_{L^{1}} \leqslant C_{2}$ independent of $y$. Then the map

$$
f \rightarrow \int_{-\infty}^{\infty} K(x, y) f(y) d y
$$

is bounded on $L^{p}, 1 \leqslant p \leqslant \infty$, with norm at most $C_{1}^{1-1 / p} C_{2}^{1 / p}$.

Proof. Using Jensen's inequality and Fubini's theorem, we find

$$
\begin{aligned}
\int_{-\infty}^{\infty}\left|\int_{-\infty}^{\infty} K(x, y) f(y) d y\right|^{p} d x & \\
& \leqslant \int_{-\infty}^{\infty} \int_{-\infty}^{\infty}|f(y) P| K(x, y) \mid d y \cdot\left(\int_{-\infty}^{\infty}|K(x, s)| d s\right)^{p-1} d x \\
& \leqslant C_{1}^{p-1} \int_{-\infty}^{\infty}\left|f(y) p \int_{-\infty}^{\infty}\right| K(x, y) \mid d x d y \leqslant C_{1}^{p-1} C_{2}\|f\|_{L^{p}}^{p}
\end{aligned}
$$

The following estimate is therefore our main tool for describing the resolvent of $L_{c}^{B}$. 
Proposition 4.4. Assume $0 \leqslant c<\frac{1}{2}, \operatorname{Re} \sqrt{\lambda+1 / 4}>\frac{1}{2}-c$ and $W(\lambda) \neq 0$. Then

(a) $\left\|K_{c}(x, \cdot, \lambda)\right\|_{L^{1}} \leqslant C(\lambda)$ independent of $x$, and

(b) $\left\|K_{c}(\cdot, y, \lambda)\right\|_{L^{1}} \leqslant C(\lambda)$ independent of $y$.

Proof. (a) Assume $x>0$ (the estimate for $x<0$ is entirely similar). We estimate the integral

$$
\int_{-\infty}^{\infty}\left|K_{c}(x, y, \lambda)\right| d y
$$

separately on the three intervals $(-\infty, 0],[0, x)$, and $[x, \infty)$. On the half line $y \leqslant 0$, and for $x>0$, we have the estimates

$$
\begin{gathered}
\left|\psi_{+}(y) \frac{\cosh y / 2}{\cosh c y}\right| \leqslant C_{1}(\lambda) e^{(\operatorname{Re} \gamma-(1 / 2-c)) y}, \\
\left|\phi_{-}(x) \frac{\cosh c x}{\cosh x / 2}\right| \leqslant C_{2}(\lambda) e^{-(\operatorname{Re} \gamma+(1 / 2-c)) x} \leqslant C_{2}(\lambda) .
\end{gathered}
$$

Then, since $x>0$, these estimates imply

$$
\int_{-\infty}^{0}\left|K_{c}(x, y, \lambda)\right| d y \leqslant \frac{C_{1} C_{2}}{|W(\lambda)|} \cdot \frac{1}{\operatorname{Re} \gamma-(1 / 2-c)} .
$$

Now $\psi_{+}$is a linear combination of $\phi_{-}$and $\phi_{+}$. So for $y>0$, we estimate

$$
\left|\psi_{+}(y) \frac{\cosh y / 2}{\cosh c y}\right| \leqslant C_{3}(\lambda) e^{(\operatorname{Re} \gamma+(1 / 2-c)) y} .
$$

Together with the estimate (3.2), this yields

$$
\int_{0}^{x}\left|K_{c}(x, y, \lambda)\right| d y \leqslant \frac{C_{2} C_{3}}{|W(\lambda)|} \cdot \frac{1}{\operatorname{Re} \gamma+(1 / 2-c)} .
$$

Finally, on the half line $y>0$, for $x>0$ we estimate

$$
\begin{aligned}
& \left|\phi_{-}(y) \frac{\cosh y / 2}{\cosh c y}\right| \leqslant C_{4}(\lambda) e^{-(\operatorname{Re} \gamma-(1 / 2-c)) y}, \\
& \left|\psi_{+}(x) \frac{\cosh c x}{\cosh x / 2}\right| \leqslant C_{5}(\lambda) e^{(\operatorname{Re} \gamma-(1 / 2-c)) x},
\end{aligned}
$$

which yields our last estimate,

$$
\int_{x}^{\infty}\left|K_{c}(x, y, \lambda)\right| d y \leqslant \frac{C_{4} C_{5}}{|W(\lambda)|} \cdot \frac{1}{\operatorname{Re} \gamma-(1 / 2-c)} .
$$

(b) We do the estimate for

$$
\int_{-\infty}^{\infty}\left|K_{c}(x, y, \lambda)\right| d x
$$

similarly. Assume that $y \geqslant 0$. Then for $x<0$, we estimate

$$
\left|\psi_{+}(x) \frac{\cosh c x}{\cosh x / 2}\right| \leqslant C_{6}(\lambda) e^{(\operatorname{Re} \gamma-(1 / 2-c)) x} .
$$


Together with (3.4), we get (since $y \geqslant 0$ )

$$
\int_{-\infty}^{0}\left|K_{c}(x, y, \lambda)\right| d x \leqslant \frac{C_{4} C_{6}}{|W(\lambda)|} \cdot \frac{1}{\operatorname{Re} \gamma-(1 / 2-c)} .
$$

The estimates (3.4) and (3.5) yield

$$
\int_{0}^{y}\left|K_{c}(x, y, \lambda)\right| d x \leqslant \frac{C_{4} C_{5}}{|W(\lambda)|} \cdot \frac{1}{\operatorname{Re} \gamma-(1 / 2-c)} .
$$

Finally, (3.2) and (3.3) imply that

$$
\int_{y}^{\infty}\left|K_{c}(x, y, \lambda)\right| d x \leqslant \frac{C_{2} C_{3}}{|W(\lambda)|} \cdot \frac{1}{\operatorname{Re} \gamma+(1 / 2-c)} .
$$

The estimate for $y<0$ is similar.

REMARK. Suppose that $0<c<\frac{1}{2}$. Then $\operatorname{Re} \sqrt{\lambda+1 / 4}>\frac{1}{2}-c$ if and only if

$$
\operatorname{Re} \lambda>-c(1-c)-\left(\frac{\operatorname{Im} \lambda}{1-2 c}\right)^{2} \text {. }
$$

We include the simple proof: $\operatorname{Re} \sqrt{z}>a>0$ when $2(\operatorname{Re} \sqrt{z})^{2}=\operatorname{Re} z+|z|>2 a^{2}$. Then

$$
(\operatorname{Re} z)^{2}+(\operatorname{Im} z)^{2}>\left(2 a^{2}-\operatorname{Re} z\right)^{2},
$$

so $(\operatorname{Im} z)^{2}>4 a^{4}-4 a^{2} \operatorname{Re} z$, and

$$
\operatorname{Re} z>a^{2}-\left(\frac{\operatorname{Im} z}{2 a}\right)^{2}
$$

Now take $z=\lambda+\frac{1}{4}, a=\frac{1}{2}-c$.

This essentially completes the determination of the resolvent set for $L_{c}^{B}$. To complete the formal proof that $\lambda$ is in the resolvent set when $\operatorname{Re} \gamma>\frac{1}{2}-c$ and $W(\lambda) \neq 0$, we need to verify that the (bounded) integral operator with kernel $K_{c}(x, y, \lambda)$ actually yields the inverse of $\lambda-L_{c}^{B}$ on a suitable domain. This verification is straightforward; we include it for completeness.

The domain of $L_{c}^{B}$ on $L^{p}$ is the set of $C^{1}$ functions $u$ with absolutely continuous first derivative such that $u, u^{\prime}$, and $u^{\prime \prime}$ are in $L^{p}$. (On $C_{u}$, require $u^{\prime}, u^{\prime \prime}$ in $C_{u}$.) Given $f$ in $L^{p}$, define

$$
G f(x)=\int_{-\infty}^{\infty} K_{c}(x, y, \lambda) f(y) d y .
$$

To show that $G$ is a right inverse for $\lambda-L_{c}^{B}$, we must show that $u=G f$ is in the domain of $L_{c}^{B}$ and that $\left(\lambda-L_{c}^{B}\right) u=f$. We may write

$$
W(\lambda) \frac{w_{1 / 2}}{w_{c}} u(x)=\psi_{+}(x) \int_{-\infty}^{x} \phi_{-} \frac{w_{1 / 2}}{w_{c}} f(y) d y+\phi_{-}(x) \int_{x}^{\infty} \psi_{+} \frac{w_{1 / 2}}{w_{c}} f(y) d y .
$$

Clearly $u(x)$ is absolutely continuous, so may be differentiated almost everywhere. We calculate

$$
\begin{aligned}
& W(\lambda)\left(\frac{w_{1 / 2}}{w_{c}} u\right)^{\prime}(x) \\
& =\psi_{+}^{\prime}(x) \int_{-\infty}^{x} \phi_{-} \frac{w_{1 / 2}}{w_{c}} f(y) d y+\phi_{-}^{\prime}(x) \int_{x}^{\infty} \psi_{+} \frac{w_{1 / 2}}{w_{c}} f(y) d y, \text { a.e. }
\end{aligned}
$$


Now it is clear that $u^{\prime}$ is absolutely continuous. One may verify that $u^{\prime}$ is in $L^{p}$ using the proof of Proposition 3.4, for $\psi_{+}^{\prime}$ and $\phi_{-}^{\prime}$ have the same asymptotic properties as $\psi_{+}$and $\phi_{-}$.

Differentiating again, we find

$$
\begin{aligned}
W(\lambda)\left(\frac{w_{1 / 2}}{w_{c}} u\right)^{\prime \prime}(x)= & -W(\lambda) \frac{w_{1 / 2}}{w_{c}} f(x)+\psi_{+}^{\prime \prime}(x) \int_{-\infty}^{x} \phi_{-} \frac{w_{1 / 2}}{w_{c}} f(y) d y \\
& +\phi_{-}^{\prime \prime}(x) \int_{x}^{\infty} \psi_{+} \frac{w_{1 / 2}}{w_{c}} f(y) d y, \text { a.e. }
\end{aligned}
$$

Since $\phi_{-}$and $\psi_{+}$are eigenfunctions of $L_{1 / 2}^{B}$, we get

$$
\left(\lambda-L_{1 / 2}^{B}\right) \frac{w_{1 / 2}}{w_{c}} u=\frac{w_{1 / 2}}{w_{c}} f, \text { a.e. }
$$

Therefore $\left(\lambda-L_{c}^{B}\right) u=f$ a.e., hence in $L^{P}$. This implies that $u$ is in the domain of $L_{c}^{B}$. So $G$ is a right inverse for $\lambda-L_{c}^{B}$ on $L^{p}$.

To show $G$ is a left inverse for $\lambda-L_{c}^{B}$ on its domain, it suffices to show that $\lambda-L_{c}^{B}$ is one to one. (For then if $u$ is in the domain, and $f=\left(\lambda-L_{c}^{B}\right) u$, we have $\left(\lambda-L_{c}^{B}\right)(G f-u)=0$, so $G f=u$.) But if $\lambda-L_{c}^{B}$ is not one-to-one, there exists $v$ in its domain with $\left(\lambda-L_{c}^{B}\right) v=0$. Then $v^{\prime \prime}$ is absolutely continuous, so $v$ is in $C^{2}$ and $L^{p}$. Since $\lambda$ is not an eigenvalue and $\operatorname{Re} \gamma>\frac{1}{2}-c$, one may show that $v$ must be zero.

REMARK. In the discussion above we needed to know that if $\lambda$ is in the resolvent set for $L_{c}^{B}$, then $(d / d x)\left(\lambda-L_{c}^{B}\right)^{-1}$ is a bounded operator on $L^{p}$. This is actually quite a general fact for the second-order systems considered in Sattinger (1976). Also, in our treatment we did not follow Sattinger in pursuing resolvent estimates asymptotically for large $|\lambda|$. Estimates such as he requires (see Sattinger (1976), Lemma 3.4(ii)) are automatically valid for the class of second order (matrix) differential operators he considers. We defer a discussion of these issues to the Appendix.

Only one fact remains to be proved in this section.

Proposition 4.5. For $0<c<\frac{1}{2}$, the eigenvalue 0 is a simple eigenvalue for $L_{c}^{B}$ on $L^{p}\left(\right.$ and $\left.C_{u}\right)$.

Proof. We must show that the associated projection operator on $L^{p}$,

$$
P_{c}=\frac{1}{2 \pi i} \int_{\Gamma}\left(\zeta-L_{c}^{B}\right)^{-1} d \zeta
$$

is one dimensional. (Here $\Gamma$ is a smooth closed curve in the region $\operatorname{Re} \gamma>\frac{1}{2}-c$ enclosing the origin.)

First, observe that the kernel of $L_{c}^{B}$ is one dimensional. (If $L_{c}^{B} u=0$, where $u$ is in the domain of $L_{c}^{B}$, then $u^{\prime \prime}$ is absolutely continuous, so $u$ is in $C^{2}$ and $L^{p}$, hence is a multiple of $\phi_{-}$.) We claim that the quasinilpotent $D_{c}$ associated with the eigenvalue zero,

$$
D_{c}=L_{c}^{B} P_{c}=\frac{1}{2 \pi i} \int_{\Gamma} \zeta\left(\zeta-L_{c}^{B}\right)^{-1} d \zeta
$$

is actually zero. This suffices to show that $P_{c}$ has one-dimensional range. 
We argue as follows: First consider the case $c=\frac{1}{2}$ on the Hilbert space $L^{2}$. The operator $L_{1 / 2}^{B}$ is then actually selfadjoint, and one obtains $D_{1 / 2}=0$ on $L^{2}$ (we cite Kato (1976), V. 3.5-6). But then we may write

$$
\begin{aligned}
D_{c} f(x) & =\frac{1}{2 \pi i} \int_{\Gamma} \zeta \int_{-\infty}^{\infty} K_{c}(x, y, \zeta) f(y) d y d \zeta \\
& =\frac{w_{c}}{w_{1 / 2}}(x) \frac{1}{2 \pi i} \int_{\Gamma} \zeta \int_{-\infty}^{\infty} K_{1 / 2}(x, y, \zeta) \frac{w_{1 / 2}}{w_{c}} f(y) d y d \zeta=0
\end{aligned}
$$

for any $f$ in $C_{0}^{\infty}(\mathbf{R})$. But $D_{c}$ is bounded on $L^{p}$, so $D_{c}=0$. Therefore, the eigenvalue 0 is simple.

5. Weighted estimates of the shock profile. The $k$-shock profile $\phi(\xi ; \varepsilon)$ obtained from Foy (1964) when $\lambda_{k}(u)$ is genuinely nonlinear is a solution of the equation

$$
\phi_{\xi}=f(\phi)-s \phi .
$$

(Here $s=\lambda_{k}\left(u_{R}\right)+\frac{1}{2} \varepsilon$, and, for convenience, $u_{R}=0=f\left(u_{R}\right)$.) For each $\varepsilon, \phi(\xi ; \varepsilon)$ is in the stable manifold at 0 for this equation. We need the following estimate, for the 1-shock profile in particular (see the end of $\$ 2$ ):

Proposition. Fix $c<\frac{1}{2}$ positive. Then there exists a constant $K$, such that if $\varepsilon$ is sufficiently small,

$$
\sup _{\xi}|\phi(\xi ; \varepsilon)| \leqslant \varepsilon K e^{-c \varepsilon \xi} .
$$

Proof. From Foy's proof, $\phi(\xi ; \varepsilon)=\frac{1}{2} \varepsilon \psi\left(\frac{1}{2} \varepsilon \xi ; \varepsilon\right)$, where

$$
\psi(x ; \varepsilon)=\left(1-\tanh \frac{1}{2} x\right) r_{k}(0)+\varepsilon \zeta(x ; \varepsilon)
$$

and

$$
\sup _{\substack{x \in \mathbf{R} \\ 0<\varepsilon \leqslant \varepsilon_{0}}}|\zeta(x ; \varepsilon)| \leqslant C .
$$

We will show that

$$
\sup _{x}|\psi(x ; \varepsilon)| \leqslant K e^{-2 c x}
$$

(independent of $\varepsilon$ ). $\psi$ satisfies the equation

$$
\psi_{x}=\Lambda^{\varepsilon} \psi+F^{\varepsilon}(\psi)
$$

where

$$
F^{\varepsilon}(\psi)=\frac{f(\varepsilon \psi / 2)-d f(0) \varepsilon \psi / 2}{(\varepsilon / 2)^{2}}
$$

and

$$
\Lambda^{\varepsilon}=\operatorname{diag}\left\{\left(\lambda_{i}(0)-s\right) / \frac{1}{2} \varepsilon, i=1, \ldots, m\right\} .
$$

Then $\Lambda_{k k}^{\varepsilon}=-1$ and $0=F^{\varepsilon}(0)=d F^{\varepsilon}(0)$. Also, $d^{2} F^{\varepsilon}(v)=d^{2} f(\varepsilon v)$.

Our proof follows a standard proof of the existence of the stable manifold based on solving an integral equation by successive approximations (Coddington and Levinson (1955), p. 330). 
In block form, write

$$
\Lambda^{\varepsilon}=\left(\begin{array}{cc}
\Lambda^{1} & 0 \\
0 & \Lambda^{2}
\end{array}\right), \quad U_{1}(\tau)=\left(\begin{array}{cc}
e^{\tau \Lambda^{1}} & 0 \\
0 & 0
\end{array}\right), \quad U_{2}(\tau)=\left(\begin{array}{cc}
0 & 0 \\
0 & e^{\tau \Lambda^{2}}
\end{array}\right),
$$

where $\Lambda^{1}$ is diagonal with negative eigenvalues, $\Lambda^{2}$ is diagonal with positive eigenvalues. Choose $c<\frac{1}{2}$ positive. Then if $\varepsilon$ is small enough, there is a $\sigma>0$ so that

$$
\begin{array}{ll}
\left|U_{1}(\tau)\right| \leqslant e^{-(2 c+\sigma) \tau}, & \text { for } \tau \geqslant 0, \\
\left|U_{2}(\tau)\right| \leqslant e^{\sigma \tau}, & \text { for } \tau \leqslant 0 .
\end{array}
$$

Fixing $a \in \mathbf{R}^{m}$, we consider the integral equation

$$
\begin{aligned}
\theta(\tau, a)= & U_{1}(\tau-T) a+\int_{T}^{\tau} U_{1}(\tau-s) F^{\varepsilon}(\theta(s, a)) d s \\
& -\int_{\tau}^{\infty} U_{2}(\tau-s) F^{\varepsilon}(\theta(s, a)) d s
\end{aligned}
$$

The following Lipschitz estimate holds for $F^{\varepsilon}$. If $\left|\theta_{1}\right|,\left|\theta_{2}\right|<\delta$, then

$$
\left|F^{\varepsilon}\left(\theta_{1}\right)-F^{\varepsilon}\left(\theta_{2}\right)\right| \leqslant \sup _{|\theta| \leqslant \delta}|d F(\theta)|\left|\theta_{1}-\theta_{2}\right| \leqslant M \delta\left|\theta_{1}-\theta_{2}\right|,
$$

where $M$ is a bound for $d^{2} f$ in a neighborhood of 0 .

Therefore, restrict $\delta$ and $a$ so that $2|a|<\delta$ and $M \delta(1 / \sigma+1 /(2 c+\sigma))<\frac{1}{2}$. We solve (5.2) by successive approximations: set $\theta^{0}(\tau, a) \equiv 0$. Then $\theta^{1}(\tau, a)=$ $U_{1}(\tau-T) a$, and

$$
\left|\theta^{1}(\tau, a)-\theta^{0}\right| \leqslant e^{-2 c(\tau-T)}|a| .
$$

By induction, we show that

$$
\left|\theta^{l+1}(\tau, a)-\theta^{l}\right| \leqslant|a| e^{-2 c(\tau-T)} \cdot 2^{-l} .
$$

Indeed,

$$
\begin{aligned}
\left|\theta^{l+1}(\tau, a)-\theta^{\prime}\right| \leqslant & \int_{T}^{\tau} e^{-(2 c+\sigma)(\tau-s)}\left(M \delta|a| e^{-2 c(s-T)} \cdot 2^{-l+1}\right) d s \\
& +\int_{\tau}^{\infty} e^{\sigma(\tau-s)}\left(M \delta|a| e^{-2 c(s-T)} \cdot 2^{-l+1}\right) d s \\
& =M \delta|a| 2^{-l+1}\left(e^{-2 c(\tau-T)} e^{-\sigma \tau} \int_{T}^{\tau} e^{\sigma s} d s+e^{\sigma \tau+2 c T} \int_{\tau}^{\infty} e^{-(2 c+\sigma) s} d s\right) \\
& \leqslant M \delta|a| e^{-2 c(\tau-T)}\left(\frac{1}{\sigma}+\frac{1}{2 c+\sigma}\right) 2^{-l+1} \leqslant|a| e^{-2 c(\tau-T)} \cdot 2^{-l} .
\end{aligned}
$$

Therefore $\theta^{\prime}$ converges uniformly to $\theta(\tau, a)$ with

$$
|\theta(\tau, a)| \leqslant 2|a| e^{-2 c(\tau-T)} \quad \text { for } \tau \geqslant T .
$$

This $\theta$ is a solution of $(5.1)$ and $\theta(T, a)$ has a special form: $\theta_{j}(T, a)=a_{j}$ if $j \leqslant k$. What is more, $\theta(\tau, a)$ is the unique solution of (5.1) with $\theta_{j}(T)=a_{j}$ for $j \leqslant k$ so $\theta \rightarrow 0$ as $\tau \rightarrow \infty$ (here we apply the stable manifold theorem). What we have shown is that the size of the neighborhood in the proof of the stable manifold theorem on 
which the estimate (5.3) holds does not shrink to zero as $\varepsilon \rightarrow 0$. Instead, this estimate holds whenever

$$
|a|<\frac{\delta}{2}<\left(4 M\left(\frac{1}{\sigma}+\frac{1}{2 c+\sigma}\right)\right)^{-1} .
$$

Now $\psi(\tau ; \varepsilon)=(1-\tanh \tau / 2) r_{k}+\varepsilon \zeta(\tau ; \varepsilon)$, where $|\zeta| \leqslant C$ independent of $\varepsilon$. Thus if $\varepsilon_{0}$ is sufficiently small, say $\varepsilon_{0}<\delta / 4 C$, then there exists a fixed $T$ so that

$$
|\psi(T ; \varepsilon)|<\delta / 2, \quad \text { for all } \varepsilon<\varepsilon_{0} .
$$

Since $\psi(\tau) \rightarrow 0$ as $\tau \rightarrow \infty$, this implies that $\psi(\tau)=\theta(\tau, \psi(T))$ by the stable manifold theorem (for $\varepsilon$ fixed). Therefore,

$$
|\psi(\tau ; \varepsilon)| \leqslant\left(\delta e^{2 c T}\right) e^{-2 c \tau}, \text { for all } \varepsilon<\varepsilon_{0} \text { and } \tau \geqslant T .
$$

Since $\psi(\tau ; \varepsilon)$ is bounded, there exists $K$ such that

$$
|\psi(\tau, \varepsilon)| \leqslant K e^{-2 c \tau}, \text { for all } \varepsilon<\varepsilon_{0} \text { and } \tau \text { real. }
$$

6. The resolvent equation for $\tilde{\mathcal{L}}_{c}^{\prime}$. We proceed to complete the proof of Theorem 2.1 by showing that if $\lambda \neq 0$ lies exterior to a suitable sector strictly contained in the left-half plane, then $\lambda$ is in the resolvent set for $\tilde{\mathcal{L}}_{c}^{\prime}$; i.e., the solution operator to the resolvent equation $\left(\tilde{\mathcal{E}}_{c}^{\prime}-\lambda\right) u=f$ is a bounded operator on $\left(L^{p}\right)_{0}^{m}\left(\right.$ or $\left.\left(C_{u}\right)_{0}^{m}\right)$. We introduce some convenient notation for the components of $\tilde{\mathcal{L}}_{c}^{\prime}$ in block form (see $\S 2$ ).

$$
\begin{array}{ll}
L_{c}^{1}=w_{c} L^{1} w_{c}^{-1}, & N_{c}^{1}=w_{c} M^{1} e^{c x}, \\
\tilde{N}_{c}=e^{-c x} \tilde{M} w_{c}^{-1}, & \tilde{L}_{c}=e^{-c x} \tilde{L} e^{c x} .
\end{array}
$$

Also set

$$
\begin{array}{ll}
L_{c}^{B}=w_{c} L^{B} w_{c}^{-1}, & R_{c}^{1} z^{1}=-w_{c}\left[R_{1}^{1} w_{c}^{-1} z^{1}\right]_{x}, \\
L_{c}^{T}=e^{-c x} L^{T} e^{c x}, & \tilde{R}_{c} \tilde{z}=-e^{-c x}\left[\left(\phi^{B} \tilde{A}+\varepsilon \tilde{R}\right) e^{c x} \tilde{z}\right]_{x} .
\end{array}
$$

Then

$$
\tilde{\varrho}_{c}^{\prime}=\left[\begin{array}{cc}
L_{c}^{B}+\varepsilon R_{c}^{1} & N_{c}^{1} \\
\tilde{N}_{c} & L_{c}^{T}+\tilde{R}_{c}
\end{array}\right] .
$$

The operators $R_{c}^{1}, \tilde{R}_{c}$, and $\tilde{N}_{c}$ are first order differential operators with smooth, uniformly bounded coefficients as $\varepsilon \rightarrow 0$. Provided $c<\frac{1}{2}$, the same is true for $N_{c}^{1}$ because of the result of the previous section,

$$
\sup _{x}\left|\psi(x ; \varepsilon) e^{2 c x}\right| \leqslant K \quad \text { independent of } \varepsilon .
$$

Consider the resolvent equation, for $\lambda \in \mathbf{C}, f \in\left(L^{p}\right)_{0}^{m}$ or $\left(C_{u}\right)_{0}^{m}$ :

$$
\left[\begin{array}{cc}
L_{c}^{B}+\varepsilon R_{c}^{1}-\lambda & N_{c}^{1} \\
\tilde{N}_{c} & L_{c}^{T}+\tilde{R}_{c}-\lambda
\end{array}\right]\left(\begin{array}{c}
z^{1} \\
\tilde{z}
\end{array}\right)=\left(\begin{array}{c}
f^{1} \\
\tilde{f}
\end{array}\right) .
$$

Fix $\beta$ with $0<\beta<c(1-c)$ and $\alpha, 0<\alpha<\pi / 2$, so that the only point in $S_{\alpha}(-\beta)$ (see §3) in the spectrum of $L_{c}^{B}$ is the point $\lambda=0$. Choose $r, 0<r<\beta$, and delete from the right-facing sector $S_{\alpha}(-\beta)$ the disk of radius $r$ centered at the origin, obtaining a region

$$
\mathscr{P}_{\alpha}=S_{\alpha}(-\beta) \backslash\{\lambda \in \mathbf{C} \| \lambda \mid \leqslant r\} \quad \text { (see Figure 2). }
$$




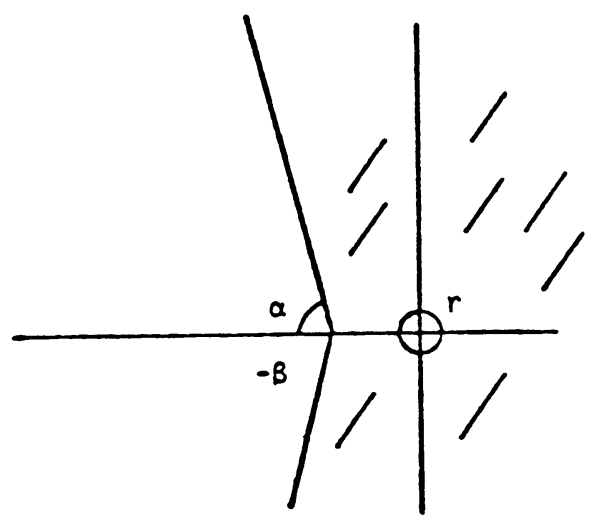

FIGURE 2. The set $\mathscr{P}_{\alpha}$

Claim. If $\varepsilon$ is sufficiently small, $\alpha<\pi / 2$ may be chosen (depending on $\varepsilon$ ) so that $\mathscr{P}_{\alpha}$ is contained in the resolvent set of $\tilde{L}_{c}^{\prime}$.

Proof. (1) There exists $C_{1}$ so that $\left\|R_{c}^{1}\left(L_{c}^{B}-\lambda\right)^{-1}\right\| \leqslant C_{1}$ for all $\lambda$ in $\mathscr{\rho}_{\alpha}$. (For this we require some asymptotic control on $\left\|\left(L_{c}^{B}-\lambda\right)^{-1}\right\|$ and $\left\|(d / d x)\left(L_{c}^{B}-\lambda\right)^{-1}\right\|$ as $|\lambda| \rightarrow \infty$ in $S_{\alpha}(-\beta)$. The necessary estimates, proved in detail on $C_{u}$, are found in the Appendix.)

(2) For $\varepsilon<1 / 2 C_{1}$, the operator

$$
L_{c}^{B}+\varepsilon R_{c}^{1}-\lambda=\left[I+\varepsilon R_{c}^{1}\left(L_{c}^{B}-\lambda\right)^{-1}\right]\left(L_{c}^{B}-\lambda\right)
$$

is invertible for all $\lambda$ in $\mathscr{P}_{\alpha}$, with norm

$$
\left\|\left(L_{c}^{1}-\lambda\right)^{-1}\right\| \leqslant 2\left\|\left(L_{c}^{B}-\lambda\right)^{-1}\right\| .
$$

(3) For $\lambda$ in $\mathscr{P}_{\alpha}$ and $\varepsilon<1 / 2 C_{1}$ we may eliminate $\tilde{N}_{c}$ from the resolvent equation, obtaining

$$
\left[\begin{array}{cc}
L_{c}^{1}-\lambda & N_{c}^{1} \\
0 & \left(I+Q\left(L_{c}^{T}-\lambda\right)^{-1}\right)\left(L_{c}^{T}-\lambda\right)
\end{array}\right]\left(\begin{array}{c}
z^{1} \\
\tilde{z}
\end{array}\right)=\left(\begin{array}{c}
f^{1} \\
\tilde{f}-\tilde{N}_{c}\left(L_{c}^{1}-\lambda\right)^{-1} f^{1}
\end{array}\right)
$$

where $Q=\tilde{R}_{c}-\tilde{N}_{c}\left(L_{c}^{1}-\lambda\right)^{-1} N_{c}^{1}$.

(4) There exists $C_{2}$ so that for all $\lambda$ in $\mathscr{P}_{\alpha}$

$$
\left\|\tilde{N}_{c}\left(L_{c}^{1}-\lambda\right)^{-1}\right\| \leqslant C_{2}
$$

(5) Apply Proposition 3.1 for each component of $L_{c}^{T}$. There exists $\varepsilon_{0}>0$ so that if $\varepsilon<\varepsilon_{0}$, we may find $\alpha<\pi / 2$ so for all $\lambda \in \mathcal{P}_{\alpha}$,

$$
\left\|N_{c}^{1}\left(L_{c}^{T}-\lambda\right)^{-1}\right\| \leqslant 1 / 4 C_{2} \text { and }\left\|Q\left(L_{c}^{T}-\lambda\right)^{-1}\right\| \leqslant 1 / 2
$$

Therefore, we may immediately solve for $\tilde{z}$, obtaining the estimate

$$
\|\tilde{z}\| \leqslant 2\left\|\left(L_{c}^{T}-\lambda\right)^{-1}\right\|\left(\|\tilde{f}\|+C_{2}\left\|f^{1}\right\|\right) .
$$

Then $z^{1}$ is determined by

$$
z^{1}=\left(L_{c}^{1}-\lambda\right)^{-1}\left(f^{1}-N_{c}^{1} \tilde{z}\right)
$$


and since

$$
\tilde{z}=\left(L_{c}^{T}-\lambda\right)^{-1} B\left(\tilde{f}-\tilde{N}_{c}\left(L_{c}^{1}-\lambda\right)^{-1} f^{1}\right),
$$

where $B=\left(I+Q\left(L_{c}^{T}-\lambda\right)^{-1}\right)^{-1}$, so $\|B\| \leqslant 2$, we find (using (5) to estimate $N_{c}^{1} \tilde{z}$ ) that

$$
\left\|z^{1}\right\| \leqslant 2\left\|\left(L_{c}^{B}-\lambda\right)^{-1}\right\|\left(\left\|f^{1}\right\|+\frac{2}{4 C_{2}}\left(\|\tilde{f}\|+C_{2}\left\|f^{1}\right\|\right)\right) .
$$

This establishes the claim above, showing the $\mathscr{P}_{\alpha}$ is in the resolvent set of $\tilde{e}_{c}^{\prime}$.

To complete the proof of Theorem 2.1 , it remains only to show that inside the disk $|\lambda| \leqslant r$, the spectrum of $\tilde{\mathcal{L}}_{c}^{\prime}$ consists solely of a simple eigenvalue at the origin. Consider two projections $P_{0}$ and $P_{\varepsilon}$ defined on $\left(L^{p}\right)_{0}^{m}$ or $\left(C_{u}\right)_{0}^{m}$ by

$$
P_{\varepsilon}=\frac{1}{2 \pi i} \int_{\Gamma}\left(\zeta-\tilde{\mathcal{L}}_{c}^{\prime}\right)^{-1} d \zeta, \quad P_{0}=\left(\begin{array}{cc}
P_{B} & 0 \\
0 & 0
\end{array}\right)
$$

in block form, where

$$
P_{B}=\frac{1}{2 \pi i} \int_{\Gamma}\left(\zeta-L_{c}^{B}\right)^{-1} d \xi
$$

Here the integration is taken in the positive sense around a circle $\Gamma$ centered at the origin with radius $\tilde{r}, r<\tilde{r}<\beta$. From the theory for $L_{c}^{B}$ in $\S 4, P_{0}$ is a one-dimensional projection.

Claim. If $\varepsilon$ is sufficiently small, then $\left\|P_{\varepsilon}-P_{0}\right\|<1$.

This implies that $P_{\varepsilon}$ is one dimensional. But $\mathcal{L}^{\prime}$, hence $\tilde{\mathcal{L}}_{c}^{\prime}$, has an eigenvalue at 0 , since $\mathcal{L}^{\prime} \phi_{\xi}=0$. The point zero is therefore the only point inside the circle of radius $r$ in the spectrum of $\tilde{\mathcal{L}}_{c}^{\prime}$.

The proof of this claim is straightforward, using the resolvent estimates we have developed. For $\lambda$ on $\Gamma, f$ in $\left(L^{p}\right)_{0}^{m}$, let

$$
\left.\left(\begin{array}{c}
z^{1} \\
\tilde{z}
\end{array}\right)=\left(\begin{array}{c}
\tilde{e}_{c}^{\prime}-\lambda \\
\tilde{u}^{\prime}
\end{array}\right), \quad \begin{array}{c}
f^{1} \\
\tilde{f}
\end{array}\right), \quad z^{B}=\left(L_{c}^{B}-\lambda\right)^{-1} f^{1} .
$$

Then it suffices to show that given $\delta>0$, then for $\varepsilon$ sufficiently small,

$$
\|\tilde{z}\|<\delta\|f\| \text { and }\left\|z^{1}-z^{B}\right\|<\delta\|f\|
$$

for all $\lambda$ on $\Gamma$. The first inequality is clearly guaranteed by our previous estimate for $\tilde{z}$ and the resolvent estimates for $L_{c}^{T}$ (Proposition 3.1). For the second, consider $z^{1}$ in more detail:

$$
\begin{aligned}
z^{1}= & \left(L_{c}^{B}-\lambda\right)^{-1}\left[I+\varepsilon R_{c}\left(L_{c}^{B}-\lambda\right)^{-1}\right]^{-1} \\
& \cdot\left[f^{1}-N_{c}^{1}\left(L_{c}^{T}-\lambda\right)^{-1} B\left(\tilde{f}-\tilde{N}_{c}\left(L_{c}^{1}-\lambda\right)^{-1} f^{1}\right)\right] .
\end{aligned}
$$

By Proposition 3.1, we can make $\left\|N_{c}^{1}\left(L_{c}^{T}-\lambda\right)^{-1}\right\|$ as small as we like by choosing $\varepsilon$ sufficiently small, and we can also achieve

$$
\left\|\left[I+\varepsilon R_{c}^{1}\left(L_{c}^{B}-\lambda\right)^{-1}\right]^{-1}-I\right\|<\frac{1}{2} \delta,
$$


from which we may extract the second estimate. So the claim holds, completing the proof of Theorem 2.1 .

Appendix. Resolvent estimates in weighted norm for certain parabolic systems. Here we develop an abstract treatment of some resolvent estimates which were required in the theory of asymptotic orbital stability of traveling waves developed by Sattinger (1976). For the class of operators and spaces with scalar weights considered by Sattinger, we show that the asymptotic estimates he required hold automatically, so need not be separately checked. Similar estimates are commonly developed for parabolic operators in unweighted spaces in the theory of fractional powers of sectorial operators, which are generators of analytic semigroups (a good reference is Henry, (1981)). Our treatment is self-contained, and proceeds in the spirit of perturbation theory for operators generating quasi-bounded semigroups. At the end of this section we prove a result involving matrix weights, validating the estimates for our operator $\mathcal{L}^{\prime}$ on the spaces $\left(C_{u}\right)_{c}^{m}$ considered in the main body of this paper.

Let us describe the estimates involved. Consider an operator

$$
L u=P u_{x x}+M(x) u_{x}+N(x) u,
$$

where $u(x) \in \mathbf{R}^{m}, P$ is a positive definite matrix, diagonal for simplicity, and $M(x)$ and $N(x)$ are bounded uniformly continuous matrix-valued functions. Let $w(x)$ be a given scalar weight function. $L$ will be considered as an operator on the weighted space

$$
B_{w}=\left\{u: \mathbf{R} \rightarrow \mathbf{R}^{m} \mid w(x) u(x) \text { is bounded and uniformly continuous }\right\},
$$

with domain

$$
\mathscr{D}(L)=\left\{u \in B_{w} \mid u_{x} \text { and } u_{x x} \text { are in } B_{w}\right\} \text {. }
$$

The space $B_{w}$ is equipped with the norm

$$
\|u\|_{w}=\max _{i} \sup _{x}\left|w(x) u^{i}(x)\right| .
$$

We also define a norm

$$
\|u\|_{w, 1}=\|u\|_{w}+\left\|u_{x}\right\|_{w} .
$$

The weight function $w(x)$ should satisfy

(i) $w(x) \geqslant 1$ for all $x$;

(ii) $\sup _{x}|1-w(x+t) / w(x)| \rightarrow 0$ as $t \rightarrow 0$.

In Sattinger's framework, $L$ was obtained by linearizing about a given traveling wave of a nonlinear parabolic system. Condition (i) above is explicit, and (ii) implicit, in Sattinger's analysis. Condition (ii) ensures that the shift $u(x) \rightarrow u(x+t)$ is a bounded operator on $B_{w}$, continuous in $t$, and implies that $w(x)$ is continuous and grows only exponentially as $|x| \rightarrow \infty$. The use of spaces of uniformly continuous functions is also implicit in Sattinger's work.

The second proposition below contains the estimate Sattinger requires in his Lemma 3.4 and Theoren 4.1. The first proposition simply states that $-L$ is a sectorial operator in the sense of Henry (1981) or $m$-sectorial in the sense of Kato (1976). 
Proposition A.1. The operator $L$ on $B_{w}$ with domain $\mathscr{D}(L)$ is a closed, densely defined operator. For some $\alpha, \beta$ real, with $0<\alpha<\pi / 2$, the sector

$$
S_{\alpha \beta}=\{\lambda \in \mathbf{C}|\operatorname{Re}(\lambda-\beta) \geqslant-\cos \alpha| \lambda-\beta \mid\}
$$

is in the resolvent set of $L$, and for any such sector $S_{\alpha \beta}$,

$$
\left\|(\lambda-L)^{-1} f\right\|_{w} \leqslant C_{\alpha \beta}\|f\|_{w} / \lambda-\beta \mid
$$

for all $\lambda \in S_{\alpha \beta}, f \in B_{w}$.

Proposition A.2. For any $\lambda$ in the resolvent set of $L, d / d x \circ(\lambda-L)^{-1}$ is a bounded operator on $B_{w}$, and for any sector $S_{\alpha \beta}$ contained in the resolvent set of $L$,

$$
\left\|(\lambda-L)^{-1} f\right\|_{w, 1} \leqslant \frac{C_{\alpha \beta}^{\prime}}{|\lambda-\beta|^{1 / 2}}\|f\|_{w}
$$

for all $\lambda \in S_{\alpha \beta}, f \in B_{w}$.

Our approach to the proofs will be as follows: First we verify (A.1) for the scalar operator $u \rightarrow u_{x x}$; then (A.1) is valid for the diagonal operator $u \rightarrow P u_{x x}$. We then establish the result for $L$ by treating the lower order terms by perturbation arguments. The same procedure is used for Proposition A.2.

Our analysis begins with a study of the translation group $U(t)$ acting on $B_{w}$ by

$$
(U(t) u)(x)=u(x+t) .
$$

LEMMA A.3. $U(t)$ is a strongly continuous, quasi-bounded group, meaning that for some constants $M$ and $b$,

$$
\|U(t) u\|_{w} \leqslant M e^{b|t|}\|u\|_{w} .
$$

The infinitesimal generator of $U(t)$ is the operator $D u=u_{x}$, with domain

$$
\mathscr{D}(D)=\left\{u \in B_{w} \mid u_{x} \in B_{w}\right\} \text {. }
$$

The resolvent set of $D$ includes the set of $\lambda \in \mathrm{C}$ with $|\operatorname{Re} \lambda|>b$, and for such $\lambda$ we have the estimate

$$
\left\|(\lambda-D)^{-1} u\right\|_{w} \leqslant M\|u\|_{w} /|\operatorname{Re} \lambda|-b .
$$

In order to carry out the proof, we first estimate the weight.

Claim. There exist constants $M$ and $b$ such that

$$
\sup _{x} w(x+t) / w(x) \leqslant M e^{b|t|} \text { for all } t .
$$

Proof. Using property (ii) satisfied by $w(x)$, we may find $\varepsilon>0$ and $b \geqslant 0$ so that

$$
\sup _{|x| \leqslant \varepsilon} \sup _{x} w(x+t) / w(x)=e^{b \varepsilon} \text {. }
$$

Given any real $t$, we may write $t=n \varepsilon+\tilde{t}$, where $n$ is an integer and $|\tilde{t}| \leqslant \varepsilon$. Since $w(x+j \varepsilon) / w(x+(j-1) \varepsilon) \leqslant e^{b \varepsilon}$ for any $j$, we obtain

$$
w(x+t) / w(x) \leqslant\left(e^{b \varepsilon}\right)^{|m|} \cdot e^{b \varepsilon} \leqslant M e^{b \mid \eta},
$$

where $M=e^{2 b \varepsilon}$. So the claim (A.5) is established. 
Proof of Lemma A.3. The claim above implies the estimate (A.3). For $u \in B_{w}$, let us verify strong continuity:

$$
\begin{aligned}
\sup _{x}|w(x)(u(x+t)-u(x))| \leqslant & \sup _{x}|(w(x)-w(x+t)) u(x+t)| \\
& +\sup _{x}|w(x+t) u(x+t)-w(x) u(x)| .
\end{aligned}
$$

The second term tends to zero as $t \rightarrow 0$ because $w u(x)$ is uniformly continuous, and the first term tends to zero because $w u(x+t)$ is bounded and

$$
\sup _{x}|1-w(x) / w(x+t)| \rightarrow 0 \quad \text { as } t \rightarrow 0 .
$$

Consider the operator $D u=u_{x}$. It is not hard to show that $\mathscr{D}(D)$ is dense in $B_{w}$. We claim that, if $\operatorname{Re} \lambda>b$, then $\lambda-D$ is invertible, with

$$
(\lambda-D)^{-1} f=\int_{0}^{\infty} e^{-\lambda t} U(t) f d t, \quad f \in B_{w} .
$$

Indeed, letting $v$ denote the right-hand side, we have

$$
\|v\|_{w} \leqslant \int_{0}^{\infty} e^{-t \operatorname{Re} \lambda} \cdot M e^{b t} d t\|f\|_{w} \leqslant \frac{M}{\operatorname{Re} \lambda-b}\|f\|_{w} .
$$

Also,

$$
v(x)=\int_{0}^{\infty} e^{-\lambda t} f(x+t) d t=e^{\lambda x} \int_{x}^{\infty} e^{-\lambda t} f(t) d t,
$$

so $v$ is differentiable and $\lambda v-v_{x}=f$, so $v$ is in the domain of $D$. Therefore $\lambda-D$ is invertible on $B_{w}$ and (A.7) holds.

When $\operatorname{Re} \lambda<-b$, a similar analysis holds with (A.7) replaced by

$$
(\lambda-D)^{-1} f=\int_{0}^{-\infty} e^{-\lambda t} U(t) f d t .
$$

The identities (A.7) and (A.8) imply that $D$ is the infinitesimal generator of the group $U(t)$, but we will not use this fact. Refer to Kato (1976) for the details.

Proof of Proposition A.1 for $D^{2}$. The domain of the operator $D^{2}$ is

$$
\mathscr{D}\left(D^{2}\right)=\left\{u \in B_{w} \mid u_{x} \text { and } u_{x x} \text { are in } B_{w}\right\} \text {. }
$$

Consider the resolvent equation for $D^{2}$ :

$$
\left(\lambda-D^{2}\right) u=f \text {. }
$$

Write $\lambda=\gamma^{2}, \operatorname{Re} \gamma \geqslant 0$. Then $\lambda-D^{2}=(\gamma-D)(\gamma+D)$, and if $\operatorname{Re} \gamma>b$, then $\pm \gamma$ is in the resolvent set of $D$, so

$$
u=\left(\lambda-D^{2}\right)^{-1} f=(\gamma+D)^{-1}(\gamma-D)^{-1} f
$$

and

$$
\|u\|_{w} \leqslant M^{2}\|f\|_{w} /(\operatorname{Re} \gamma-b)^{2} .
$$

So the resolvent set of $D^{2}$ includes the region $\operatorname{Re} \sqrt{\lambda}>b$, which is "exterior" to the parabola $\operatorname{Re} \sqrt{\lambda}=b$. This region contains a sector $S_{\alpha \beta}$, where $\beta>b^{2}, 0<\alpha<\pi / 2$. Let $S_{\alpha \beta}$ now denote any sector in the resolvent set of $D^{2}$, with $0<\alpha<\pi / 2$. We seek 
to show (A.1) holds. The resolvent is uniformly bounded in any compact subset of $S_{\alpha \beta}$, so we need only verify (A.1) for $|\lambda|$ sufficiently large. It suffices to prove the following

Claim. There exist constants $C$ and $c$ so that if $\lambda \in S_{\alpha \beta}$ and $|\lambda| \geqslant C$, then

$$
(\operatorname{Re} \sqrt{\lambda}-b)^{2} \geqslant c|\lambda-\beta| \text {. }
$$

For the proof, first choose $\tilde{\alpha}, 0<\tilde{\alpha}<\alpha$, and pick $C>2|\beta|$ so large that if $\lambda \in S_{\alpha \beta}$ and $|\lambda|>C$, then $\lambda \in S_{\tilde{\alpha} 0}$, i.e., $\arg \lambda \leqslant \pi-\tilde{\alpha}$. Since the set $\operatorname{Re} \sqrt{\lambda}=2 b$ is a parabola, we may inflate $C$ so that if $\lambda \in S_{\alpha \beta}$ and $|\lambda|>C$, then $\operatorname{Re} \sqrt{\lambda}>2 b$. We also have $\arg \sqrt{\lambda} \leqslant(\pi-\tilde{\alpha}) / 2$, so it follows that $(\operatorname{Re} \sqrt{\lambda}-b) /|\sqrt{\lambda}|$ has a positive infimum $c_{1}$ for $\lambda \in S_{\alpha \beta},|\lambda|>C$. Then

$$
(\operatorname{Re} \sqrt{\lambda}-b)^{2} \geqslant c_{1}|\lambda|
$$

and since $C>2|\beta|,|\lambda| \geqslant c_{3}|\lambda-\beta|$ for $|\lambda|>C, c_{3}>0$, and the claim follows. Hence Proposition A.1 is established for the operator $D^{2}$.

Now Proposition A.1 is valid for $p D^{2}, p>0$, by a simple scaling argument. We may separately analyze each component of the resolvent equation for $P D^{2}$, $\left(\lambda-P D^{2}\right) u=f$, where $P$ is a positive definite diagonal matrix, and find that Proposition A.1 is valid for this operator as well.

Our treatment of the lower order terms of $L$ is based on the notion of relative boundedness of closed operators, and on a Landau-Kolmogorov inequality for generators of bounded semigroups (a recent reference is Chernoff, 1979).

Definition (cf. Kato (1976)). Let $A$ and $B$ be operators on a Banach space, with $\mathscr{Q}(B) \supset \mathscr{D}(A) . B$ is said to be $A$-bounded with bound $c_{0}$ if for any $c>c_{0}$, there exists $C$ so that for all $v \in \mathscr{D}(A)$,

$$
\|B v\| \leqslant C\|v\|+c\|A v\| \text {. }
$$

Lemma A.4. Let $M$ and $b$ be given from Lemma A.3. Then for any $u \in \mathscr{D}\left(D^{2}\right)$,

$$
\|(D-b) u\|_{w} \leqslant \frac{M}{\lambda}\left\|(D-b)^{2} u\right\|_{w}+(M+1) \lambda\|u\|_{w} \quad \text { for all } \lambda>0,
$$

$$
\|(D-b) u\|_{w}^{2} \leqslant C_{0}\left\|(D-b)^{2} u\right\|_{w}\|u\|_{w} \text { for some constant } C_{0} .
$$

COROllary. $D$ is $D^{2}$-bounded with bound 0 on $B_{w}$.

Proof. For $\lambda>0$,

$$
\left\|(\lambda-(D-b))^{-1}\right\| \leqslant M / \lambda .
$$

For $u \in \mathscr{D}\left(D^{2}\right)$, we may write

$$
(\lambda+D-b) u=(\lambda-(D-b))^{-1}\left(\lambda^{2}-(D-b)^{2}\right) u
$$

so

$$
\|(D-b) u\|_{w} \leqslant \lambda\|u\|_{w}+\frac{M}{\lambda}\left(\lambda^{2}\|u\|_{w}+\left\|(D-b)^{2} u\right\|_{w}\right),
$$

yielding (A.9). Put $\lambda=\left(\left\|(D-b)^{2} u\right\|_{w} /\|u\|_{w}\right)^{1 / 2}$ to obtain (A.10). For the corollary argue as follows: $D$ is $(D-b)$-bounded, since

$$
\|D u\|_{w} \leqslant\|(D-b) u\|_{w}+b\|w\|_{w} .
$$


By (A.9), $(D-b)$ is $(D-b)^{2}$-bounded with bound 0 . Finally, $(D-b)^{2}$ is $D^{2}$ bounded, for

$$
\begin{aligned}
\left\|(D-b)^{2} u\right\|_{w} & \leqslant\left\|D^{2} u\right\|_{w}+2 b\|(D-b) u\|_{w}+b^{2}\|u\|_{w} \\
& \leqslant\left\|D^{2} u\right\|_{w}+2 b\left(\varepsilon\left\|(D-b)^{2} u\right\|_{w}+C(\varepsilon)\|u\|_{w}\right)+b^{2}\|u\|_{w} .
\end{aligned}
$$

Taking $\varepsilon$ sufficiently small, we find

$$
\left\|(D-b)^{2} u\right\|_{w} \leqslant c\left\|D^{2} u\right\|_{w}+C\|u\|_{w} .
$$

Then $D$ is clearly $D^{2}$-bounded with bound 0 .

Proof of Proposition A.1. Let $L u=P u_{x x}+M(x) u_{x}+N(x) u$, and let $L_{0} u=$ $P u_{x x}, B u=M(x) u_{x}+N(x) u$. Since $M(x)$ and $N(x)$ are bounded matrices, $B$ is $D$-bounded, i.e.,

$$
\|B u\|_{w} \leqslant c\left\|u_{x}\right\|_{w}+C\|u\|_{w} \text { for } u \in \mathscr{D}(D) .
$$

Applying the previous corollary, $B$ is $D^{2}$-bounded with bound 0 , hence $L_{0}$-bounded with bound 0 . But then $L_{0}$ is $L$-bounded, for

$$
\left\|L_{0} u\right\|_{w} \leqslant\|L u\|_{w}+\|B u\|_{w} \leqslant\|L u\|_{w}+\varepsilon\left\|L_{0} u\right\|_{w}+C(\varepsilon)\|u\|_{w},
$$

and if $\varepsilon<1$ we obtain $\left\|L_{0} u\right\|_{w} \leqslant c\|L u\|_{w}+C\|u\|_{w}$. Hence $B$ is $L$-bounded with bound 0 .

Fix a sector $S_{\alpha \beta}$ with $0<\alpha<\pi / 2$. If $\lambda \in S_{\alpha \beta}$ and $|\lambda|$ is sufficiently large, $\lambda-L_{0}$ is invertible and (A.1) holds for $L_{0}$. Rewrite (A.1) in the equivalent form,

$$
\|u\|_{w} \leqslant \frac{C_{\alpha \beta}}{|\lambda-\beta|}\|(\lambda-L) u\|_{w} \quad \text { for } u \in \mathscr{D}(L) .
$$

We claim that (A.12) holds for $\lambda$ in $S_{\alpha \beta}$ if $|\lambda|$ is sufficiently large. For $u \in \mathscr{D}(L)$,

$$
\begin{aligned}
\left\|\left(\lambda-L_{0}\right) u\right\|_{w} & \leqslant\|(\lambda-L) u\|_{w}+\|B u\|_{w} \leqslant\|(\lambda-L) u\|_{w}+\varepsilon\|L u\|_{w}+C(\varepsilon)\|u\|_{w} \\
& \leqslant(1+\varepsilon)\|(\lambda-L) u\|_{w}+(|\lambda| \varepsilon+C(\varepsilon))\|u\|_{w} .
\end{aligned}
$$

Now (A.12) holds for $L_{0}$ for some constant $C_{\alpha \beta}$. Fix $\varepsilon<1 / 4 C_{\alpha \beta}$. Then if $|\lambda|$ is sufficiently large,

$$
\frac{C_{\alpha \beta}}{|\lambda-\beta|}(|\lambda| \varepsilon+C(\varepsilon))<\frac{1}{2},
$$

and we have

$$
\|u\|_{w} \leqslant \frac{C_{\alpha \beta}}{|\lambda-\beta|}\|(\lambda-L) u\|_{w}+\frac{1}{2}\|u\|_{w}
$$

for $|\lambda|$ sufficiently large, yielding (A.12). So Proposition A.1 is established.

Proof of Proposition A.2. Define the operators $L, L_{0}$, and $B$ as above. Let us show that if $\lambda$ is in the resolvent set of $L$, then $D(\lambda-L)^{-1}$ is a bounded operator on $B_{w}$. Observe that since $D$ is $D^{2}$-bounded with bound 0 , and $L_{0}$ is $L$-bounded, it 
follows that $D$ is $L$-bounded with bound 0 . Then, since range $(\lambda-L)^{-1}=\mathscr{D}(L) \subset$ $\mathscr{D}(D)$, we have, for any $f \in B_{w}$,

$$
\begin{aligned}
\left\|D(\lambda-L)^{-1} f\right\|_{w} & \leqslant c\left\|L(\lambda-L)^{-1} f\right\|_{w}+C\left\|(\lambda-L)^{-1} f\right\|_{w} \\
& \leqslant c\|f\|_{w}+(c|\lambda|+C)\left\|(\lambda-L)^{-1} f\right\|_{w} \leqslant C(\lambda)\|f\|_{w} .
\end{aligned}
$$

Let us now assume that (A.2) holds for the operator $L_{0}$ for $|\lambda|$ sufficiently large, $\lambda$ in any given sector $S_{\alpha \beta}, 0<\alpha<\pi / 2$, and show that it holds for $L$ if $|\lambda|$ is sufficiently large. Rewrite (A.1) in the equivalent form,

$$
\|u\|_{w}+\|D u\|_{w} \leqslant \frac{C_{\alpha \beta}^{\prime}}{|\lambda-\beta|^{1 / 2}}\|(\lambda-L) u\|_{w},
$$

for $u \in \mathscr{D}(L), \lambda \in S_{\alpha \beta},|\lambda|$ sufficiently large. Now

$$
\left\|\left(\lambda-L_{0}\right) u\right\|_{w} \leqslant\|(\lambda-L) u\|_{w}+\|B u\|_{w} \leqslant\|(\lambda-L) u\|_{w}+C(\|u\|+\|D u\|) .
$$

Since (A.13) is assumed to hold for $L_{0}$, we therefore have

$$
\left(\|u\|_{w}+\|D u\|_{w}\right)\left(1-\frac{C_{\alpha \beta}^{\prime} C}{|\lambda-\beta|^{1 / 2}}\right) \leqslant \frac{C_{\alpha \beta}^{\prime}}{|\lambda-\beta|^{1 / 2}}\|(\lambda-L) u\|_{w} .
$$

Therefore, if $|\lambda|$ is sufficiently large, (A.13) holds for $L$ as well, perhaps with a larger constant $C_{\alpha \beta}^{\prime}$.

The last step in our proof is to establish (A.13) for the operator $D^{2}$. By considering each component separately, it will follow immediately that (A.13) is valid for the diagonal operator $L_{0}$. Our approach is to use the Landau-Kolmogorov inequality (A.10), along with the sectorial estimate (A.12) for $D^{2}$.

Fix any sector $S_{\alpha \beta}$. Then for $\lambda$ in this sector, $|\lambda|$ sufficiently large, $\lambda-D^{2}$ is invertible and (A.12) holds. Recall from (A.11) that $(D-b)^{2}$ is $D^{2}$-bounded. Using (A.10), we find that for $u \in \mathscr{D}\left(D^{2}\right)$,

$$
\|(D-b) u\|_{w}^{2} \leqslant C_{0}\|u\|_{w}\left(c\left\|D^{2} u\right\|_{w}+C\|u\|_{w}\right) .
$$

Now $\left\|D^{2} u\right\|_{w} \leqslant\left\|\left(\lambda-D^{2}\right) u\right\|_{w}+|\lambda|\|u\|_{w}$, so, estimating $\|u\|_{w}$ via (A.12), we obtain

$$
\|(D-b) u\|_{w}^{2} \leqslant \frac{C_{0} C_{\alpha \beta}}{|\lambda-\beta|}\left(c+(c|\lambda|+C) \frac{C_{\alpha \beta}}{|\lambda-\beta|}\right)\left\|\left(\lambda-D^{2}\right) u\right\|_{w}^{2} .
$$

Therefore, for $|\lambda|$ sufficiently large,

$$
\|(D-b) u\|_{w} \leqslant \frac{\tilde{C}_{\alpha \beta}}{|\lambda-\beta|^{1 / 2}}\left\|\left(\lambda-D^{2}\right) u\right\|_{w} .
$$

Now $D$ is $(D-b)$-bounded, and we have

$$
\|u\|_{w}+\|D u\|_{w} \leqslant\|(D-b) u\|_{w}+(b+1)\|u\|_{w} .
$$

Using (A.14) and (A.12), we obtain (A.13) for $D^{2}$ for all $\lambda \in S_{\alpha \beta}$ with $|\lambda|$ sufficiently large. This concludes the proof of Proposition A.2.

We conclude this section with some brief remarks about matrix-valued weight functions which show that our operator $\mathcal{L}^{\prime}$ of (1.6) satisfies the estimates (A.1) and (A.2), at least on the spaces $\left(C_{u}\right)_{\varepsilon c / 2}^{m}$ for $\varepsilon$ sufficiently small. Consider a smooth 
matrix function $W(x)$ which is diagonal for all $x$ for simplicity, with $W_{i i}(x)>0$ for each $i$. Define the weighted space

$$
B_{W}=\left\{u: \mathbf{R} \rightarrow \mathbf{R}^{m} \mid W u(x) \text { is bounded and uniformly continuous }\right\},
$$

with norm

$$
\|u\|_{W}=\max _{i} \sup _{x}\left|W_{i i}(x) u_{i}(x)\right| .
$$

For example, if $W_{11}(x)=\cosh c x$ and $W_{j j}(x)=e^{-c x}$ for $j>1$, then $B_{W}=\left(C_{u}\right)_{c}^{m}$. The theory of this appendix does not apply in $B_{W}$; e.g., multiplication by a constant, nondiagonal matrix $M$ need not be a bounded operator in $B_{W}$. However, in special circumstances the estimates (A.1) and (A.2) may hold if the weight $W(x)$ may be used to define a similarity transformation which takes the operator under consideration to a "nice" operator acting on the unweighted space $\left(C_{u}\right)^{m}$.

Let $L$ be an operator on $B_{W}$,

$$
L u=P u_{x x}+M(x) u_{x}+N(x) u,
$$

where $P$ is a positive definite diagonal matrix, and $M$ and $N$ are smooth bounded matrix-valued functions. Define an operator on $\left(C_{u}\right)^{m}$ by

$$
L_{W} v=W L W^{-1} v=P v_{x x}+\tilde{M}(x) v_{x}+\tilde{N}(x) v .
$$

Now $\tilde{M}(x)$ and $\tilde{N}(x)$, in general, are not bounded functions of $x$, but suppose they are. (This is the case of interest in this paper, where $L_{W}$ corresponds to $\tilde{L}_{c}^{\prime}$, cf. (2.4).) Then the results of this appendix apply for $L_{W}$ on the space $\left(C_{u}\right)^{m}$, yielding (A.1) and (A.2) for $L_{W}$. Consider the equivalent formulations (A.12) and (A.13). Then (A.12) holds immediately for $L$ on $B_{W}$. Given any $u \in \mathscr{D}(L)$, then $v=W u$ is in $D\left(L_{W}\right)$, and

$$
\|u\|_{W}=\|v\|_{\infty} \leqslant \frac{C_{\alpha \beta}}{|\lambda-\beta|}\left\|\left(\lambda-W L W^{-1}\right) v\right\|_{\infty}=\frac{C_{\alpha \beta}}{|\lambda-\beta|}\|(\lambda-L) u\|_{W} .
$$

To verify (A.13), observe that

$$
W D u=D v-W_{x} W^{-1} v .
$$

Require that $\sup _{x}\left|W_{x} W^{-1}(x)\right| \leqslant C<\infty$. (This is valid for the weights we have used in this paper.) Then

$$
\begin{aligned}
\|u\|_{W}+\|D u\|_{W} & \leqslant(1+C)\|v\|_{\infty}+\|D v\|_{\infty} \leqslant \frac{C_{\alpha \beta}^{\prime}(1+C)}{|\lambda-\beta|^{1 / 2}}\left\|\left(\lambda-L_{W}\right) v\right\|_{\infty} \\
& =\frac{C_{\alpha \beta}^{\prime \prime}}{|\lambda-\beta|^{1 / 2}}\|(\lambda-L) u\|_{W} .
\end{aligned}
$$

Supplement. Marginal stability on unweighted spaces. Here we show, using Theorem 1.1, that the linearized operator $\mathcal{L}^{\prime}$ for the weak 1-shock profile has no unstable eigenvalues. More precisely and more generally

THEOREM. For $\varepsilon>0$ sufficiently small, the resolvent set of $\mathcal{L}^{\prime}$ on the unweighted space $\left(C_{u}\right)^{m}\left(\right.$ also $\left.\left(L^{p}\right)^{m}\right)$ includes all complex $\lambda$ exterior to the sector $S_{\alpha}\left(-\frac{1}{4} \varepsilon^{2} \beta\right)$ of Theorem 1.1 and exterior to some parabolic region $P_{C}=\left\{\lambda \mid \operatorname{Re} \lambda \leqslant-C(\operatorname{Im} \lambda)^{2}\right\}$ which lies in the left-half plane but includes the origin. 
For the proof, it is enough to show that $L^{\prime}$ has no eigenvalues in the region described. This is due to a characterization of the essential spectrum (spectrum aside from isolated points of finite multiplicity) that may be found in Henry (1981). Let $A_{+}=d f\left(u_{R}\right)-s, A_{-}=d f\left(u_{L}\right)-s$, and form the sets

$$
S_{ \pm}=\left\{\lambda \in \mathbf{C} \mid \operatorname{det}\left(-\tau^{2}-i \tau A_{ \pm}-\lambda\right)=0 \text { for some real } \tau\right\} .
$$

Then $S_{+} \cup S_{-}$is contained in the essential spectrum, but the connected component of $\mathbf{C} \backslash\left(S_{+} \cup S_{-}\right)$which is unbounded to the right is devoid of essential spectrum. Note that here, any $\lambda \in S_{ \pm}$is of the form

$$
\operatorname{Re} \lambda=-\tau^{2}, \quad \operatorname{Im} \lambda=\tau\left(\lambda_{j}\left(u_{R, L}\right)-s\right) \text { for some } j=1, \ldots, m .
$$

so $S_{+} \cup S_{-}$is contained in a parabolic region $\left\{\lambda \in \mathrm{C} \mid \operatorname{Re} \lambda \leqslant-C(\operatorname{Im} \lambda)^{2}\right\}$ for some $C>0$. Now suppose $\lambda \in \mathbf{C} \backslash\left(S_{\alpha}\left(-\frac{1}{4} \varepsilon^{2} \beta\right) \cup P_{c}\right)$ and $\lambda$ is an eigenvalue, so $\left(\mathcal{L}^{\prime}-\lambda\right) \Psi$ $=0$ for some function $\Psi(x)$ in $\operatorname{Dom}\left(\mathcal{L}^{\prime}\right)$. (Therefore $\Psi$ is bounded and smooth.) It will be convenient not to scale the independent variable, so for $0 \leqslant c<\frac{1}{2}$, we let

$$
\mathcal{L}_{c}^{\prime}=W_{c} \varrho^{\prime} W_{c}^{-1}=\left[\begin{array}{cc}
L_{c}^{1} & M_{c}^{1} \\
\tilde{M}_{c} & \tilde{L}_{c}
\end{array}\right], \quad W_{c}(x)=\left[\begin{array}{cc}
\cosh \varepsilon c x / 2 & 0 \\
0 & e^{-\varepsilon c x / 2}
\end{array}\right] .
$$

We claim that $W_{c} \Psi$ is bounded, so that $\lambda$ is an eigenvalue of $\mathcal{L}_{c}^{\prime}$, contradicting Theorem 1.1.

Step 1. We claim $\left|e^{-\varepsilon c x / 2} \Psi(x)\right|<$ const, $x \leqslant 0$. We apply standard theorems on asymptotic behavior of solutions of linear ODE's (Coddington and Levinson 1955). As $x \rightarrow-\infty, \Psi$ is asymptotic to a solution of $u_{x x}-A_{-} u_{x}-\lambda u=0$, i.e.,

$$
\Psi(x)=e^{\mu_{-} x}(r+o(1)) \text { and } \Psi^{\prime}(x)=\mu_{-} e^{\mu_{-} x}(r+o(1)),
$$

where $\left(\mu_{-}^{2}-\mu_{-} A_{-}-\lambda\right) r=0$ and $\operatorname{Re} \mu_{-} \geqslant 0$. In this case, $\mu_{-}$is of the form $2 \mu_{-}=$ $\left(\lambda_{j}\left(u_{L}\right)-s\right)+\left(\left(\lambda_{j}\left(u_{L}\right)-s\right)^{2}+\lambda\right)^{1 / 2}$ for some $j$. For $\lambda$ "exterior" to $S_{-}$, we have $\operatorname{Re}\left(\left(\lambda_{j}\left(u_{j}\right)-s\right)^{2}+\lambda\right)^{1 / 2}>\lambda_{j}\left(u_{L}\right)-s$, so $\operatorname{Re} \mu_{-} \geqslant \lambda_{1}\left(u_{L}\right)-s$. Recall that $s=$ $\lambda_{1}\left(u_{R}\right)+\frac{1}{2} \varepsilon, \nabla \lambda_{1} \cdot r_{1}\left(u_{R}\right)=1$, and $u_{L}=u_{R}+\varepsilon r_{1}+O\left(\varepsilon^{2}\right)$, so $\operatorname{Re} \mu_{-} \geqslant \frac{1}{2} \varepsilon-O\left(\varepsilon^{2}\right)$. For $\varepsilon$ small, we then must have $\operatorname{Re} \mu_{-}-\varepsilon c / 2>0$, so the claim holds.

Step 2. We claim $\left|e^{\varepsilon c x / 2} \Psi^{1}\right|<$ const, $x \geqslant 0$. A different argument is needed here. In block form, $\Psi=\left(\Psi^{1}, \tilde{\Psi}\right)$, and

$$
\left(L_{c}^{1}-\lambda\right)\left[\cosh \varepsilon c x / 2 \Psi^{1}\right]=-M_{c}^{1}\left[e^{-\varepsilon c x / 2} \tilde{\Psi}\right] .
$$

The right side is bounded, from Step 1. By the construction of the resolvent of $\tilde{L}_{c}^{\prime}$ in $\S 6, \lambda$ is in the resolvent set of $L_{c}^{1}$ if $\lambda \neq 0$ is exterior to $S_{\alpha}\left(-\varepsilon^{2} \beta / 4\right)$. Therefore we may find $\Phi$ in $\operatorname{Dom}\left(L_{c}^{1}\right)$, so bounded, with

$$
\left(L_{c}^{1}-\lambda\right) \Phi=-M_{c}^{1}\left[e^{-\varepsilon c x / 2} \tilde{\Psi}\right]
$$

Hence

$$
\left(L_{0}^{1}-\lambda\right)\left[\Psi^{1}-\operatorname{sech} \varepsilon c x / 2 \Phi\right]=0 .
$$

The expression in brackets is bounded, so has asymptotic behavior $e^{\mu_{+} x}(1+o(1))$ as $x \rightarrow+\infty$, where $\left(\right.$ since $\left.\lambda_{1}\left(u_{R}\right)-s=-\varepsilon / 2\right)$

$$
\mu_{+}^{2}+\mu_{+} \varepsilon / 2-\lambda=0, \quad \operatorname{Re} \mu_{+} \leqslant 0,
$$


so $\mu_{+}=-\varepsilon / 4-\left((\varepsilon / 4)^{2}+\lambda\right)^{1 / 2}$. Since $\lambda$ is exterior to $S_{+}, \operatorname{Re} \mu_{+}<-\varepsilon / 2$, so $\operatorname{Re} \mu_{+}+\varepsilon c / 2<0$, and the claim follows.

ACKNOWLEDGement. This author wishes to express great thanks to Professor Andrew Majda for his inspiration and guidance throughout the course of this work.

\section{REFERENCES}

P. Chernoff, Optimal Landau-Kolmogorov inequalities for dissipative operators in Hilbert and Banach spaces, Advances in Math. 34 (1979), 137-144.

E. Coddington and N. Levinson, Theory of ordinary differential equations, McGraw-Hill, New York, 1955.

J. Conlon, $A$ theorem in ordinary differential equations with an application to hyperbolic conservation laws, Advances in Math. 35 (1980), 1-18.

L. Foy, Steady state solutions of conservation laws with viscosity terms, Comm. Pure Appl. Math. 17 (1964), 177-188.

D. Henry, Geometric theory of semilinear parabolic equations, Lecture Notes in Math., Vol. 840, Springer-Verlag, New York, 1981.

A. M. Il'in and O. A. Oleinik, Asymptotic behavior of solutions of the Cauchy problem for some quasilinear equations for large time, Mat. Sb. 56 (93) (1960), 191-216.

T. Kato, Perturbation theory for linear operators (2nd ed.), Springer-Verlag, New York, 1976; (1st ed.), 1966.

P. Lax, Hyperbolic systems of conservation laws. II, Comm. Pure Appl. Math. 10 (1957), 537-566.

L. A. Peletier, Asymptotic stability of travelling waves, in Instability of Continuous Systems, (IUTAM Symposium, 1969), H. Leipholz, ed., Springer, New York, 1971, pp. 418-422.

D. H. Sattinger, On the stability of waves of nonlinear parabolic systems, Advances in Math. 22 (1976), $312-355$.

Mathematics Research Center, University of Wisconsin, Madison, Wisconsin 53706

Current address: Department of Mathematics, University of Michigan, Ann Arbor, Michigan 48109 